\title{
The geometry and topology of toric hyperkähler manifolds
}

\author{
Roger Bielawski ANd ANDREW S. DANCER ${ }^{1}$
}

\begin{abstract}
We study hyperkähler manifolds that can be obtained as hyperkähler quotients of flat quaternionic space by tori, and in particular, their relation to toric varieties and Delzant polytopes. When smooth, these hyperkähler quotients are complete. We also show that for smooth projective toric varieties $X$ the cotangent bundle of $X$ carries a hyperkähler metric, which is complete only if $X$ is a product of projective spaces. Our hyperkähler manifolds have the homotopy type of a union of compact toric varieties intersecting along toric subvarieties. We give explicit formulas for the hyperkähler metric and its Kähler potential.
\end{abstract}

\section{Introduction.}

A $4 n$-dimensional manifold is hyperkähler if it possesses a Riemannian metric $g$ which is Kähler with respect to three complex structures $J_{1}, J_{2}, J_{3}$ satisfying the quaternionic relations $J_{1} J_{2}=-J_{2} J_{1}=J_{3}$ etc. To date the most powerful technique for constructing such manifolds is the hyperkähler quotient method of Hitchin, Karlhede, Lindström and Roček [HKLR]. The power of this method lies in the fact that a flat hyperkähler space may have highly nontrivial quotients.

In this paper we shall make a detailed study of a class of hyperkähler quotients of flat quaternionic space $\mathbb{H}^{d}$ by subtori of $T^{d}$. The geometry of these spaces turns out to be closely connected with the theory of toric varieties, that is, varieties of complex dimension $n$ admitting an action of $\left(\mathbb{C}^{*}\right)^{n}$ with an open dense orbit. The toric varieties we shall be concerned with have a Kähler metric preserved by the action of $T^{n} \leq\left(\mathbb{C}^{*}\right)^{n}$.

If $4 n$ is the dimension of our hyperkähler quotient there is an isometric action of $T^{n}$ which is holomorphic with respect to all the complex structures. We shall refer to our manifolds as toric hyperkähler manifolds (cf. [Go]).

\footnotetext{
${ }^{1}$ Supported in part by NSERC grant OGP0184235
} 
We shall study various topological and metric properties of toric hyperkähler manifolds. First we give necessary and sufficient conditions for a hyperkähler quotient $M$ of quaternionic space by our torus actions to be smooth (Theorem 3.2) or an orbifold (Theorem 3.3). When smooth, $M$ is complete as a Riemannian manifold. We show that the hyperkähler moment map $\phi$ for the induced torus action on $M$ is a surjection onto $\mathbb{R}^{3 n}$ with connected fibers. This can be viewed as an analogue of the convexity theorem for compact toric varieties. We also explain how to read off the singular orbits and fixed points of the $T^{n}$ action (Theorem 3.1).

Our discussion is influenced by the work of Delzant [De] and Guillemin [Gu1],[Gu2], who have shown that a large class of toric varieties can be obtained as Kähler quotients of $\mathbb{C}^{d}$ by subtori of $T^{d}$. A guiding principle of our work is that, while a compact Kähler toric variety is determined by a convex polytope, a complete toric hyperkähler orbifold is determined by an arrangement of affine subspaces.

In section 4 we discuss how the existence of a large family of compact 3-Sasakian manifolds found by Boyer, Galicki and Mann [BGM 1],[BGMR] can be read off from our results.

In section 5 we show that the generic complex structure of a toric hyperkähler orbifold is that of an affine variety (Theorem 5.1). In section 6 we discuss the topology of toric hyperkähler orbifolds $M$, and show that it depends only the torus used to obtain $M$ and not on the moment map (Theorem 6.1). We identify the homotopy type of our orbifolds as that of a union of finitely many toric varieties intersecting along toric subvarieties (Theorem 6.5). We also give a combinatorial formula for the Betti numbers of toric hyperkähler orbifolds (Theorem 6.7).

If $X$ is a toric variety arising from Delzant's construction, we show in section 7 that the cotangent bundle $T^{*} X$ carries a natural hyperkähler metric whose restriction to the zero section is the Kähler metric on $X$. This hyperkähler metric is complete only when $X$ is a product of projective spaces. We also discuss when the metric on $T^{*} X$ can be smoothly completed.

The last two sections deal with the Kähler geometry of our manifolds. We give an explicit formula for the Kähler form (Theorem 8.3), generalizing the formula of Guillemin [Gu1] for compact toric varieties. We also give an explicit description of the Riemannian metric (Theorem 9.1), which corresponds to finding a solution of generalized Bogomolny equations of Pedersen and Poon [PP].

We refer the reader to [HKLR] for a thorough discussion of Kähler and hyperkähler quotients. Let us remark here that a particular class of our manifolds was studied by Goto [Go] (see Remark 3.6). Even for this class 
our point of view is different from Goto's as we particularly stress the relation with algebraic toric varieties. There is also some relation with the work of Nakajima [Na].

\section{Toric varieties.}

In this section we shall give a quick overview of Kähler quotients of $\mathbb{C}^{d}$ by tori and in particular of Delzant's construction of certain toric varieties from polytopes [De]. We follow the exposition of Guillemin [Gu1],[Gu2].

The real torus $T^{d}=\left\{\left(t_{1}, \ldots, t_{d}\right) \in \mathbb{C}^{d}:\left|t_{i}\right|=1\right\}$ acts diagonally on $\mathbb{C}^{d}$ preserving the flat Kähler metric whose Kähler form is

$$
\frac{\sqrt{-1}}{2} \sum_{k=1}^{d} d z_{k} \wedge d \bar{z}_{k} .
$$

The moment map for this action is

$$
\mu(z)=\frac{1}{2} \sum_{k=1}^{d}\left|z_{k}\right|^{2} e_{k}+c,
$$

where the $e_{i}$ are the standard basis vectors of $\mathbb{R}^{d}$ and $c$ is an arbitrary constant in $\mathbb{R}^{d}$. If $N$ is a subtorus of $T^{d}$ whose Lie algebra $\mathfrak{n} \subset \mathbb{R}^{d}$ is generated by rational vectors, then we can perform the Kähler quotient construction with respect to $N$. Such a subtorus is determined by a collection of nonzero integer vectors $\left\{u_{1}, \ldots, u_{d}\right\}$ (which we shall always take to be primitive) generating $\mathbb{R}^{n}$. For then we obtain exact sequences of vector spaces

$$
\begin{gathered}
0 \longrightarrow \mathfrak{n} \stackrel{\imath}{\longrightarrow} \mathbb{R}^{d} \stackrel{\beta}{\longrightarrow} \mathbb{R}^{n} \longrightarrow 0 \\
0 \longrightarrow \mathbb{R}^{n} \stackrel{\beta^{*}}{\longrightarrow} \mathbb{R}^{d} \stackrel{\imath^{*}}{\longrightarrow} \mathfrak{n}^{*} \longrightarrow 0,
\end{gathered}
$$

where the map $\beta$ sends $e_{i}$ to $u_{i}$. There is a corresponding exact sequence of groups

$$
1 \rightarrow N \rightarrow T^{d} \rightarrow T^{n} \rightarrow 1 .
$$

In order to obtain a smooth Kähler quotient one has to make certain assumptions on $N$. We will not discuss these in full generality (but see below for the case when the $u_{i}$ come from a polytope). In the next section we shall 
give necessary and sufficient conditions for the corresponding hyperkähler quotient to be smooth.

The torus $N$ acts on $\mathbb{C}^{d}$ preserving the Kähler form (2.1), and the moment map for $N$ is, from (2.2),

$$
\mu(z)=\frac{1}{2} \sum_{k=1}^{d}\left|z_{k}\right|^{2} \alpha_{k}+c,
$$

where $\alpha_{k}=\imath^{*}\left(e_{k}\right)$. The constant $c$ is of the form

$$
c=\sum_{k=1}^{d} \lambda_{k} \alpha_{k}
$$

for some scalars $\lambda_{1}, \ldots, \lambda_{k} \in \mathbb{R}$. If 0 is a regular value of the moment map (2.6), we obtain a smooth Kähler quotient $X=\mu^{-1}(0) / N$ which is a toric variety. The torus $T^{n}=T^{d} / N$ of (2.5) acts on $X$ and gives rise to a moment map $\phi: X \rightarrow \mathbb{R}^{n}$. If $X$ is compact, the image of this map is a convex polytope $\Delta$ called the Delzant polytope of $X$. (Note that its vertices are not required to lie on an integer lattice and in this respect the Delzant polytope differs from the Newton polytope of algebraic toric varieties).

Conversely, any smooth compact toric variety $X$ of complex dimension $n$, with a Kähler metric invariant under $T^{n} \leq\left(\mathbb{C}^{*}\right)^{n}$, comes from Delzant's construction. For the $T^{n}$ action induces a moment map as above, whose image is a convex polytope $\Delta$ in $\mathbb{R}^{n}$. The smoothness of $X$ corresponds to the properties that precisely $n$ edges meet at each vertex of $\Delta$ (that is, $\Delta$ is simple), and that the directions of these $n$ edges are given by a $\mathbb{Z}$-basis of $\mathbb{Z}^{n}$. The polytope $\Delta$ is defined by a system of inequalities of the form

$$
\left\langle x, u_{i}\right\rangle \geq \lambda_{i}, \quad(i=1, \ldots, d),
$$

where $u_{i}$ is the inward-pointing normal vector to the $i$-th $(n-1)$-dimensional face of $\Delta$. Now $X$ is produced by the Kähler quotient construction described above, where the vectors $u_{i}$ and the scalars $\lambda_{i}$ are those in (2.8).

The Kähler quotient $X=\mu^{-1}(0) / N$ can be identified as follows with the quotient of an open subset of $\mathbb{C}^{d}$ by the complexified torus $N^{\mathbb{C}}$. Every orbit in $\mathbb{C}^{d}$ of $\left(T^{d}\right)^{\mathbb{C}}$ is of the form

$$
\mathbb{C}_{I}^{d}=\left\{\left(z_{1}, \ldots, z_{d}\right): z_{i}=0 \text { iff } i \notin I\right\}
$$

for some multi-index $I=\left(i_{1}, \ldots, i_{r}\right), 1 \leq i_{1}<\ldots<i_{r} \leq d$ (we allow $r=0$ ). If $F$ is a face of $\Delta$ of codimension $r$, then $F$ is defined by replacing 
the inequalities of (2.8) by equalities for $i$ belonging to the complement of some multi-index $I$ of length $d-r$. If we let $\mathbb{C}_{F}^{d}=\mathbb{C}_{I}^{d}$ then the set

$$
\mathbb{C}_{\Delta}^{d}=\bigcup_{F} \mathbb{C}_{F}^{d}
$$

is open, and $X$ is biholomorphic to $\mathbb{C}_{\Delta}^{d} / N^{\mathbb{C}}$. (Note that to be consistent with the notation later in this paper our definition of $\mathbb{C}_{I}^{d}$ is dual to that of Guillemin).

Example 2.1. Consider the following $n+1$ vectors in $\mathbb{R}^{n}: u_{i}=e_{i}, 1 \leq i \leq$ $n$, and $u_{n+1}=-\left(e_{1}+\ldots+e_{n}\right)$. For any negative scalars $\lambda_{1}, \ldots, \lambda_{n+1}$, the polytope $\Delta$ defined by (2.8) is similar to the standard simplex in $\mathbb{R}^{n}$ (see Fig.1 for $n=2)$. Here $\mathbb{C}_{\Delta}^{d}=\mathbb{C}^{n+1}-\{0\}$ and $N^{\mathbb{C}}$ is the diagonal $\mathbb{C}^{*}$, so $X$ is $\mathbb{C} P^{n}$.

Example 2.2. In this example the vectors $u_{i}$ are not determined by a polytope. We take $u_{1}=-e_{1}, u_{2}=u_{3}=e_{1}$ in $\mathbb{R}$ and $\lambda_{1}=-1, \lambda_{2}=\frac{1}{2}$ and $\lambda_{3}=0$. This time $\mathfrak{n}$ is spanned by $(1,1,0)$ and $(1,0,1)$ and the zero set of the moment map (2.6) is described by the equations: $\left|z_{1}\right|^{2}+\left|z_{2}\right|^{2}=1,-\left|z_{2}\right|^{2}+\left|z_{3}\right|^{2}=1$. In this case $\left(\mathbb{C}^{d}\right)^{\min }=\left(\mathbb{C}^{2}-\{0\}\right) \times \mathbb{C}^{*}$ and $X$ is $\mathbb{C} P^{1}$.

If we fix $u_{1}, \ldots, u_{d}$, the resulting variety still depends on the choice of the moment map, that is, on the scalars $\lambda_{i}$. In particular the topology may change when we pass through a critical value of $c=\sum \lambda_{k} \alpha_{k}$. This change corresponds to a proper birational morphism of the toric varieties ([Od,Gu2]).

Example 2.3. Consider the vectors $u_{1}=e_{1}, u_{2}=e_{2}, u_{3}=-e_{1}, u_{4}=$ $-a e_{1}-e_{2}$ in $\mathbb{R}^{2}$, where $a$ is a positive integer. Figure 2 shows the case $a=1$. For large $\lambda_{3}$ the polytope $\Delta$ is a trapezoid and the corresponding surface $X$ is the Hirzebruch surface $\mathbb{P}(O \oplus O(a))$. Moving the line orthogonal to $u_{3}$ beyond the intersection point of lines orthogonal to $u_{2}$ and $u_{4}$ corresponds to blowing down the divisor $D$ with $D \cdot D=-a$. The blown-down surface is the weighted projective space $\mathbb{C} P^{2}(1,1, a)$, which is nonsingular only for $a=1$.

A toric variety is also determined by a fan $\mathcal{F}$, that is, a collection of rational strongly convex polyhedral cones in $\mathbb{R}^{n}$ such that each face of a cone in $\mathcal{F}$ is also a cone in $\mathcal{F}$ and the intersection of two cones in $\mathcal{F}$ is a face of each $[\mathrm{Fu}]$. A convex polytope $\Delta$ described by (2.8) determines a fan 
$\mathcal{F}$ as follows: the cone $\left\{\sum_{i \in I} t_{i} u_{i}: t_{i} \geq 0\right\}$ belongs to $\mathcal{F}$ if and only if the $(n-1)$-dimensional faces of $\Delta$ corresponding to $u_{i}, i \in I$, meet in $\Delta$. The passage from a polytope to the fan is equivalent to forgetting the Kähler metric of $X$.

\section{Toric hyperkähler manifolds.}

We shall now discuss hyperkähler quotients of $\mathbb{H}^{d}$ by subtori of $T^{d}$. The quaternionic vector space $\mathbb{H}^{d}$ is a flat hyperkähler manifold with complex structures $J_{1}, J_{2}, J_{3}$ given by right multiplication by $i, j, k$. The real torus $T^{d}$ acts on $\mathbb{H}^{d}$ by left diagonal multiplication, preserving the hyperkähler structure. If we choose one complex structure, say $J_{2}$, and identify $\mathbb{H}^{d}$ with $\mathbb{C}^{d} \times \mathbb{C}^{d}$, then the action can be written as

$$
t \cdot(z, w)=\left(t \cdot z, t^{-1} \cdot w\right) .
$$

On the other hand, taking the complex structure $J_{1}$ identifies $\mathbb{H}^{d}$ with $T^{*} \mathbb{C}^{d}$, with the natural torus action induced from that on $\mathbb{C}^{d}$.

The three moment maps $\mu_{1}, \mu_{2}, \mu_{3}$ corresponding to the complex structures can be written as

$$
\mu_{1}(z, w)=\frac{1}{2} \sum_{k=1}^{d}\left(\left|z_{k}\right|^{2}-\left|w_{k}\right|^{2}\right) e_{k}+c_{1},
$$

$$
\left(\mu_{2}+\sqrt{-1} \mu_{3}\right)(z, w)=\sum_{k=1}^{d}\left(z_{k} w_{k}\right) e_{k}+c_{2}+\sqrt{-1} c_{3},
$$

where $c_{1}, c_{2}, c_{3}$ are arbitrary constant vectors in $\mathbb{R}^{d}$. Notice, that unlike in the Kähler case, the hyperkähler moment map $\left(\mu_{1}, \mu_{2}, \mu_{3}\right)$ is surjective for any choice of $c_{1}, c_{2}, c_{3}$, and in fact gives a homeomorphism $\mathbb{H}^{d} / T^{d} \rightarrow \mathbb{R}^{3 d}$.

Now, let $u_{i}(i=1, \ldots, d)$, define a subtorus $N$ of $T^{d}$ by (2.3) and (2.5). As before we assume that the vectors $u_{i}$ are integer, primitive and generate $\mathbb{R}^{n}$. The moment maps for the action of $N$ are (cf. (2.6))

$$
\begin{gathered}
\mu_{1}(z, w)=\frac{1}{2} \sum_{k=1}^{d}\left(\left|z_{k}\right|^{2}-\left|w_{k}\right|^{2}\right) \alpha_{k}+c_{1}, \\
\left(\mu_{2}+\sqrt{-1} \mu_{3}\right)(z, w)=\sum_{k=1}^{d}\left(z_{k} w_{k}\right) \alpha_{k}+c_{2}+\sqrt{-1} c_{3} .
\end{gathered}
$$


The constants $c_{j}$ are of the form

$$
c_{j}=\sum_{k=1}^{d} \lambda_{k}^{j} \alpha_{k}, \quad(j=1,2,3) .
$$

where $\lambda_{k}^{j} \in \mathbb{R}$. We shall adopt the notation

$$
\lambda_{k}=\left(\lambda_{k}^{1}, \lambda_{k}^{2}, \lambda_{k}^{3}\right), \quad(k=1, \ldots, d) .
$$

We shall denote the hyperkähler quotient $\mu^{-1}(0) / N$ corresponding to $\underline{u}=$ $\left(u_{1}, \ldots, u_{d}\right)$ and $\underline{\lambda}=\left(\lambda_{1}, \ldots, \lambda_{d}\right)$ by $M(\underline{u}, \underline{\lambda})$, or sometimes just $M$.

It will be important to consider the hyperplanes in $\mathbb{R}^{n}$

$$
H_{k}^{j}=\left\{y \in \mathbb{R}^{n}:\left\langle y, u_{k}\right\rangle=\lambda_{k}^{j}\right\}, \quad(j=1,2,3, \quad k=1, \ldots, d)
$$

and the codimension 3 flats (affine subspaces) in $\mathbb{R}^{3 n}$

$$
H_{k}=H_{k}^{1} \times H_{k}^{2} \times H_{k}^{3} \text {. }
$$

It is these flats, rather than the intersection of half-spaces as for toric varieties, that determine the structure of toric hyperkähler manifolds.

The action of $T^{n}=T^{d} / N$ on $M(\underline{u}, \underline{\lambda})$ preserves the hyperkähler structure and gives rise to a hyperkähler moment map $\phi=\left(\phi_{1}, \phi_{2}, \phi_{3}\right)$. The following result describes its essential properties.

Theorem 3.1. Let $u_{1}, \ldots, u_{d} \in \mathbb{Z}^{n}$ be primitive and span $\mathbb{R}^{n}$ and let $\lambda_{1}, \ldots, \lambda_{d} \in \mathbb{R}^{3}$. Then:

(i) The hyperkähler moment map $\phi: M \rightarrow \mathbb{R}^{3 n}$ for the action of $T^{n}$ defines a homeomorphism $M / T^{n} \rightarrow \mathbb{R}^{3 n}$. Therefore $M$ is connected.

(ii) If $x \in \mathbb{R}^{3 n}$, then the $T^{n}$-stabiliser of a point in $\phi^{-1}(x)$ is the torus whose Lie algebra is spanned by the vectors $u_{k}$ for which $x \in H_{k}$.

Proof. We claim that $(z, w)$ is in the zero set of (3.3) if and only if there exist $a \in \mathbb{R}^{n}, b \in \mathbb{C}^{n}$ such that

$$
z_{k} w_{k}+\lambda_{k}^{2}+\sqrt{-1} \lambda_{k}^{3}=\left\langle b, u_{k}\right\rangle, \quad \frac{1}{2}\left(\left|z_{k}\right|^{2}-\left|w_{k}\right|^{2}\right)+\lambda_{k}^{1}=\left\langle a, u_{k}\right\rangle
$$

for $k=1, \ldots, d$. (The first inner product is complex). Indeed the complex equation (3.3b) means that the real and imaginary parts of $\sum_{k=1}^{d}\left(z_{k} w_{k}+\right.$ $\left.\lambda_{k}^{2}+\sqrt{-1} \lambda_{k}^{3}\right) e_{k}$ are in $\operatorname{Ker} \imath^{*}$, which from (2.4) equals $\operatorname{Im} \beta^{*}$. Now

$$
\beta^{*}(s)=\sum_{k=1}^{d}\left\langle s, u_{k}\right\rangle e_{k},
$$


yielding the first equation of (3.6). The same argument works for $\mu_{1}$.

As remarked after equation (3.2), the moment map for the action of $T^{d}$ on $\mathbb{H}^{d}$ defines a homeomorphism from $\mathbb{H}^{d} / T^{d}$ onto $\mathbb{R}^{3 d}$. Since the vectors $u_{k}$ generate $\mathbb{R}^{n},(3.6)$ shows that the map $(z, w) \mapsto(a, b)$ gives a homeomorphism of the quotient by $T^{d}$ of the zero-set of (3.3) onto $\mathbb{R}^{3 n}$. We therefore obtain a homeomorphism of $M / T^{n}$ onto $\mathbb{R}^{3 n}$. We see from (2.4) that $(a, b)$ is the value of $\phi$ at the point in $M$ with representative $(z, w)$, so we have proved (i).

The $T^{n}$-stabiliser of the point in $M$ represented by $(z, w)$ is just the quotient of the $T^{d}$-stabiliser of $(z, w)$ by the $N$-stabiliser of $(z, w)$. Now $z_{k}=w_{k}=0$ if and only if both $\left\langle a, u_{k}\right\rangle=\lambda_{k}^{1}$ and $\left\langle b, u_{k}\right\rangle=\lambda_{k}^{2}+\sqrt{-1} \lambda_{k}^{3}$, that is, if and only if $(a, b) \in H_{k}$. Therefore the $T^{d}$-stabilizer of $(z, w)$ is the subtorus of $T^{d}$ whose Lie algebra is generated by the vectors $e_{k}$ for which $(a, b) \in H_{k}$. Part (ii) of the theorem now follows from (2.3).

This result shows at once that, even if $u_{k}, \lambda_{k}^{1}$ define a polytope $\Delta$ by (2.8) corresponding to a toric variety $X$, our manifold $M(\underline{u}, \underline{\lambda})$ need not be $T^{n}$-equivariantly diffeomorphic to $T^{*} X$. We can see this by considering the fixed points of $T^{n}$ on $T^{*} X$. For the fixed points of $T^{n}$ on $X$ correspond to the vertices of $\Delta$ and are therefore isolated. It follows that these are the only fixed points of $T^{n}$ on $T^{*} X$. If, however, some $n$ faces of $\Delta$ corresponding to linearly independent $u_{i}$ meet outside $\Delta$, then we get additional fixed points of $T^{n}$ on $M(\underline{u}, \underline{\lambda})$.

We shall see in section 6 that $M(\underline{u}, \underline{\lambda})$ is typically not homeomorphic to $T^{*} X$, even non-equivariantly. This is essentially due to the fact that the hyperkähler moment map $\phi: M \rightarrow \mathbb{R}^{3 n}$ is surjective, unlike in the Kähler case treated in $\S 2$, where the image of the Kähler moment map $\phi$ is a polytope in $\mathbb{R}^{n}$. This difference between the Kähler and hyperkähler picture will be important at several points in the paper.

We shall now give necessary and sufficient conditions for $\mu^{-1}(0) / N$ to be smooth or an orbifold. We shall assume that the flats are distinct.

Theorem 3.2. Suppose we are given primitive vectors $u_{1}, \ldots, u_{d} \in \mathbb{Z}^{n}$ generating $\mathbb{R}^{n}$ and elements $\lambda_{1}, \ldots, \lambda_{d}$ of $\mathbb{R}^{3}$ such that the flats $H_{k}$ are distinct. Then the hyperkähler quotient $M(\underline{u}, \underline{\lambda})$ is smooth if and only if every $n+1$ flats among the $H_{k}$ have empty intersection and whenever some $n$ flats $H_{k_{1}}, \ldots, H_{k_{n}}$ have nonempty intersection, then the set $\left\{u_{k_{1}}, \ldots, u_{k_{n}}\right\}$ is a $\mathbb{Z}$-basis for $\mathbb{Z}^{n}$.

Theorem 3.3. With the assumptions of Theorem 3.2 $M(\underline{u}, \underline{\lambda})$ is an orb- 
ifold, with at worst abelian quotient singularities, if and only if every $n+1$ flats among the $H_{k}$ have empty intersection.

Proof. (a). We begin by noting that if $J$ is a maximal set of indices satisfying $\bigcap_{k \in J} H_{k} \neq \emptyset$, then the set $\left\{u_{k}: k \in J\right\}$ spans $\mathbb{R}^{n}$. For if $t \notin J$, then by maximality $\bigcap_{k \in J \cup\{t\}} H_{k}$ is empty, so $u_{t}$ is in the span of $\left\{u_{k}: k \in J\right\}$. As we always suppose that the set of all $u_{k}$ spans $\mathbb{R}^{n}$, the claim follows.

Now we consider the following statements:

1) for all $x \in \mathbb{R}^{3 n}$, the set $\left\{u_{k}: x \in H_{k}\right\}$ is contained in a $\mathbb{Z}$-basis for $\mathbb{Z}^{n}$,

2) for all $x \in \mathbb{R}^{3 n}$, the set $\left\{u_{k}: x \in H_{k}\right\}$ is linearly independent.

We claim that 1) is equivalent to the condition of Theorem 3.2 and 2) to that of Theorem 3.3. It is obvious that 1) and 2) imply the respective conditions. Conversely, let $x \in \mathbb{R}^{3 n}$ and let $I$ be the set of indices $k$ such that $x \in H_{k}$. Let $J$ be a maximal element of the set of indices containing $I$ and satisfying $\bigcap_{k \in J} H_{k} \neq \emptyset$. By the observation made at the beginning of the proof, the set $\left\{u_{k}: k \in J\right\}$ spans $\mathbb{R}^{n}$ and in particular $\# J \geq n$. The claim now easily follows.

(b). Next, we shall show that 1),2) are equivalent to the action of $N$ on the zero level set of $\mu$ being free or locally free respectively.

Let $(z, w) \in \mu^{-1}(0)$ and let $(a, b) \in \mathbb{R}^{n} \times \mathbb{C}^{n}$ be $\phi(z, w)$, as in (3.6). We also regard $(a, b)$ as a point $x \in \mathbb{R}^{3 n}$ in the obvious way. We observe from 3.1(i) that any $(a, b) \in \mathbb{R}^{n} \times \mathbb{C}^{n}$, and hence any $x \in \mathbb{R}^{3 n}$, can occur in (3.6).

If $I=\left\{k: x \in H_{k}\right\}$, we let $\mathbb{R}_{I}^{d}$ denote the span of $\left\{e_{k}: k \in I\right\}$, and $T_{I}$ be the associated subtorus of $T^{d}$. The proof of Theorem 3.1(ii) shows that $T_{I}$ is the stabilizer of $(z, w)$ for the $T^{d}$ action.

The work of Delzant and Guillemin now shows that 1),2) are equivalent to $N \cap T_{I}$ always being trivial or finite respectively. For example, notice that $\mathfrak{n} \cap \mathbb{R}_{I}^{d}$ is zero if and only if the kernel of $\beta$ on $\mathbb{R}_{I}^{d}$ is zero, that is, if and only if the set $\left\{u_{k}: k \in I\right\}=\left\{u_{k}: x \in H_{k}\right\}$ is linearly independent.

(c). Standard results of symplectic geometry show that freeness or local freeness of the action of $N$ on $\mu^{-1}(0)$ imply that the quotient is smooth or an orbifold respectively. In both cases, $\mu^{-1}(0)$ is smooth.

We shall now show the necessity of the condition of Theorem 3.3. Suppose that $M(\underline{u}, \underline{\lambda})$ is an orbifold and let $J$ be a maximal set of indices satisfying $\bigcap_{k \in J} H_{k} \neq \emptyset$. Therefore $\left\{u_{k}: k \in J\right\}$ spans $\mathbb{R}^{n}$ and $\bigcap_{k \in J} H_{k}$ is a point, say $x$.

It follows from Theorem 3.1 that $m=\phi^{-1}(x)$ is fixed by $T^{n}$. Since $M$ is an orbifold, it has a well defined tangent space at $m$ of the form $\mathbb{R}^{4 n} / \Gamma$ 
for some finite linear group $\Gamma$, and pulling back to $\mathbb{R}^{4 n}$ we obtain a linear representation of $T^{n}$ with a finite kernel. (The dimension of $M$ must be $4 n$ because of 3.1(i).) As the $T^{n}$ action preserves the hyperkähler structure, we see that we have the standard representation of $T^{n}$ as the maximal torus in $S p(n)$.

Moreover some $T^{n}$-invariant neighbourhood of $m$ is $T^{n}$-equivariantly homeomorphic to a neighbourhood of zero in $\mathbb{R}^{4 n} / \Gamma$. Theorem 3.1(ii) now shows that \#J $\leq n$, establishing the necessity of the condition of Theorem 3.3.

In particular, if $M$ is a manifold then the condition of 3.3 holds and hence the action of $N$ on $\mu^{-1}(0)$ is locally free, so, as mentioned above, the zero set of $\mu$ is smooth. As the (quaternionic) action of $N$ is generically free, smoothness of $M$ now implies that the action of $N$ on $\mu^{-1}(0)$ is free. From above, we have now shown the necessity of the condition of Theorem 3.2.

Remark 3.4. It follows that for any fixed set of vectors $u_{k}$, the hyperkähler quotient $M(\underline{u}, \underline{\lambda})$ is an orbifold for a generic choice of vectors $\lambda_{k}$. On the other hand, this quotient is a manifold for a generic choice of vectors $\lambda_{k}$ if and only if any set of $n$ independent vectors among the $u_{i}$ is a $\mathbb{Z}$-basis for $\mathbb{Z}^{n}$. Furthermore, if the latter condition is satisfied, then the set of $\lambda_{k}$ for which $M(\underline{u}, \underline{\lambda})$ is singular has codimension 3 in $\mathbb{R}^{3 d}$ and hence the set of $\lambda_{k}$ for which $M(\underline{u}, \underline{\lambda})$ is smooth is path-connected. Therefore we expect the topology of smooth $M(\underline{u}, \underline{\lambda})$ to be independent of the vectors $\lambda_{k}$. We shall show in section 6 that this is indeed the case.

Theorems 3.1 and 3.3 imply

Corollary 3.5. Suppose that $M(\underline{u}, \underline{\lambda})$ is an orbifold (with all $H_{k}$ distinct). Then:

(i) the set of fixed points for the action of $T^{n}$ is finite and in one-to-one correspondence with the set of intersection points of $n$ among the flats.

(ii) if $x \in \mathbb{R}^{3 n}$ lies in exactly $r$ flats, then the $T^{n}$-stabiliser of a point in $\phi^{-1}(x)$ is an $r$-dimensional torus.

If the condition of Theorem 3.2 or Theorem 3.3 is satisfied, we shall refer to $M(\underline{u}, \underline{\lambda})$ as a toric hyperkähler manifold or toric hyperkähler orbifold respectively. In the former case it is a complete $4 n$-dimensional Riemannian manifold with a hyperkähler action of $T^{n}$. Not all hyperkähler manifolds with such an action can be obtained as a hyperkähler quotient of $\mathbb{H}^{d}$ by a 
torus. Examples are provided by the Taub-NUT metric and various higherdimensional analogues (see [HKLR] for the Roček metrics, and [GR] for some more recent constructions). This is a consequence of the fact that $T^{n}$ is not the only maximal abelian group preserving the hyperkähler structure of $\mathbb{H}^{n}$.

Remark 3.6. Goto [Go] considers a special class of hyperkähler quotients of $\mathbb{H}^{d}$ by tori. In his case $n=m_{1}+m_{2}+\ldots+m_{k}, d=n+k$ and the $u_{i}$ are the vectors $e_{i}$ of the standard basis of $\mathbb{R}^{n}$ together with the $k$ vectors $-\sum_{i=1}^{s_{j}} e_{i}$, $s_{j}=m_{1}+m_{2}+\ldots+m_{j}, j=1, \ldots, k$. For this class of toric hyperkähler manifolds Goto obtains statements essentially equivalent to Corollary 3.5 and Theorems 3.2 and 6.5. On the other hand, Nakajima [Na] studies very general properties of a class of quotients of flat quaternionic spaces by unitary groups. In the abelian case, his class of subtori of $T^{d}$, while larger than that of Goto, is still quite special - when $n=2$, for instance, it does not include tori from Example 2.3 for $a \neq 0,1$.

As examples of toric hyperkähler manifolds, consider the hyperkähler quotients corresponding to examples 2.1 and 2.2. In the first case we obtain the Calabi metric [Ca] on $T^{*} \mathbb{C} P^{n}$, while the second case yields the Gibbons-Hawking metric on the resolution of the Kleinian singularity $\mathbb{C}^{2} / \mathbb{Z}_{3}$ $[\mathrm{GH}, \mathrm{Hi}, \mathrm{Kr}]$.

The following example illustrates the dependence of $M(\underline{u}, \underline{\lambda})$ on the arrangement of flats (3.5) and not on the intersection of half-spaces (2.8)

Example 3.7. Let $n=2$ and $u_{1}=e_{1}, u_{2}=e_{2}, u_{3}=-e_{1}+e_{2}$. For negative scalars $\lambda_{1}, \lambda_{2}, \lambda_{3}$ with $\lambda_{2}>\lambda_{1}+\lambda_{3}$ the intersection of half-spaces (2.8) is illustrated in Figure 3. The corresponding toric variety is the line bundle $O(1)$ over $\mathbb{C} P^{1}$. Now consider the hyperkähler orbifold $M(\underline{u}, \underline{\lambda})$ with the same $u_{k}$ and $\lambda_{k}^{1}=\lambda_{k}, \lambda_{k}^{2}=\lambda_{k}^{3}=0$. Figure 4 shows the hyperplanes $H_{k}^{1} \quad(k=1,2,3)$. This is the same hyperplane arrangement as for the projective space $\mathbb{C} P^{2}$ (see Fig. 1). In fact $M(\underline{u}, \underline{\lambda})$ is $T^{*} \mathbb{C} P^{2}$ because the hyperkähler quotient of $\mathbb{H}^{3}$ by $N=\left\{(t,-t, t): t \in S^{1}\right\}$ is the same as that by $\left\{(t, t, t): t \in S^{1}\right\}$.

\section{Compact 3-Sasakian manifolds.}

We shall briefly discuss how the ideas of the previous sections can be used to produce a large family of compact 3-Sasakian manifolds considered in [BGM1,2] and [BGMR]. We recall here that 3-Sasakian manifolds are a 
special class of Einstein manifolds with positive scalar curvature. Also, a 3Sasakian manifold admits a locally free action of $S p(1)$, and the quotient is a quaternionic Kähler orbifold. A Riemannian manifold $(\mathcal{S}, g)$ is 3-Sasakian if and only if the Riemannian cone $C(\mathcal{S})=\left(\mathbb{R}^{+} \times \mathcal{S}, d r^{2}+r^{2} g\right)$ is hyperkähler.

Theorem 4.1. Let $\underline{u}=\left(u_{1}, \ldots, u_{d}\right)$ be a collection of mutually nonparallel primitive vectors in $\mathbb{Z}^{n}$ generating $\mathbb{R}^{n}$. Then the hyperkähler quotient $M(\underline{u}, \underline{0})$ is the Riemannian cone over a compact 3-Sasakian manifold $\mathcal{S}=\mathcal{S}(\underline{u})$ if and only if the following two conditions hold:

(i) every subset of $\underline{u}$ with $n$ elements is linearly independent,

(ii) every subset of $\underline{u}$ with less than $n$ elements is a part of a $\mathbb{Z}$-basis of $\mathbb{Z}^{n}$.

Proof. Let us first show that these conditions are necessary and sufficient for $M(\underline{u}, \underline{0})$ to have only one singularity, the point corresponding to $z=w=0$. From the proof of Theorems 3.1 and 3.2 it follows that $(z, w) \in \mathbb{H}^{d}$ will yield a singular point of $M(\underline{u}, \underline{0})$ precisely when there exists $(a, b) \in \mathbb{R}^{n} \times \mathbb{C}^{n}$, such that $z_{k} w_{k}=\left\langle b, u_{k}\right\rangle,\left|z_{k}\right|^{2}-\left|w_{k}\right|^{2}=2\left\langle a, u_{k}\right\rangle$ for $k=1, \ldots, d$, and the set $\left\{u_{k}:\left\langle b, u_{k}\right\rangle=\left\langle a, u_{k}\right\rangle=0\right\}$ is not a part of a $\mathbb{Z}$-basis of $\mathbb{Z}^{n}$. Assumption (ii) means that this can only happen if this set has at least $n$ elements, but assumption (i) implies that in this case $a=b=0$ and so $z=w=0$. Necessity of (i),(ii) follows easily from Theorem 3.2.

Now we recall that $\mathbb{H}^{d}$ is the Riemannian cone over the standard sphere $S^{4 d-1}$ and $S^{4 d-1}$ is a 3-Sasakian manifold. The 3-Sasakian structure of $S^{4 d-1}$ is given by the right diagonal action of $S p(1)$ on $\mathbb{H}^{d}$. Since we have chosen all $\lambda_{i}$ to be zero, the zero-set of the moment map (3.3) is invariant under the action of both $\mathbb{R}^{+}$and $S p(1)$. As the action of $N$ commutes with that of $\mathbb{R}^{+}$, and as the only singularity is at the origin, $M(\underline{u}, \underline{0})$ is a Riemannian cone over a manifold $\mathcal{S}$. The action of $S p(1)$ also commutes with $N$, and induces an action on $\mathcal{S}$ defining a 3-Sasakian structure. Finally $\mathcal{S}$ is compact since $M(\underline{u}, \underline{0})$ is complete (as a stratified manifold) and the cone is complete only if its base is. This implies that $\mathcal{S}$ is complete and so compact by Myers's theorem. Alternatively we could realize $\mathcal{S}$ as the 3-Sasakian quotient [BGM2] of $S^{4 d-1}$.

Remark 4.2. Usually the $S p(1)$ action on $\mathcal{S}=\mathcal{S}(\underline{u})$ has many different orbit types and so the quotient of $\mathcal{S}$ by $S p(1)$ is only a quaternionic-Kähler orbifold. It is a manifold only when $N$ is the circle acting diagonally on $\mathbb{H}^{n+1}$, which gives the homogenous quaternionic-Kähler manifold $\mathrm{Gr}_{2}\left(\mathbb{C}^{n+1}\right)$. 
Remark 4.3. For $n=1$ the conditions of Theorem 4.1 are void. When $n=2$ the conditions are satisfied if each pair of the vectors $u_{k}$ is linearly independent and each $u_{k}$ has relatively prime coordinates. The resulting quaternionic-Kähler orbifolds are 4-dimensional and admit an action of $T^{2}$.

\section{Complex structures.}

We shall now describe the generic complex structure of our toric hyperkähler orbifolds.

Theorem 5.1. Let $M=M(\underline{u}, \underline{\lambda})$ be a toric hyperkähler orbifold and suppose that every $n+1$ flats $H_{k}^{2} \times H_{k}^{3}$ have empty intersection in $\mathbb{R}^{2 n}$. Then $M$, equipped with the complex structure $J_{1}$, is biholomorphic to the affine variety Spec $A[W]^{N^{c}}$ where $W \subset \mathbb{C}^{d} \times \mathbb{C}^{d} \times \mathbb{C}^{n}$ is defined by the equations

$$
z_{k} w_{k}=\left\langle b, u_{k}\right\rangle-\left(\lambda_{k}^{2}+\sqrt{-1} \lambda_{k}^{3}\right), \quad(k=1, \ldots, d),
$$

and $N^{\mathbb{C}}$ acts on $\mathbb{C}^{d} \times \mathbb{C}^{d} \times \mathbb{C}^{n}$ by $t \cdot(z, w, b)=\left(t \cdot z, t^{-1} \cdot w, b\right)$.

Proof. By (3.6), the variety $W$ is precisely the zero-set of the complex moment map (3.3b). We have to show that the action of $N^{\mathbb{C}}$ on $W$ has at most discrete stabilizers and that each $N^{\mathbb{C}}$-orbit meets the zero-set of the moment map $\mu_{1}$. This will prove that the variety $W$ is smooth (since $W$ is the zero-set of the moment map for the complex-symplectic $N^{\mathbb{C}}$ action) and that $M$, the Kähler quotient of $W$ by $N$, can be identified with the complex quotient $W / N^{\mathbb{C}}$. The argument we use is a slight modification of the one used for the construction of toric varieties as Kähler quotients (see [Gu2]).

Let $(z, w) \in \mathbb{C}^{d} \times \mathbb{C}^{d}$. Then the image of the $N^{\mathbb{C}}$-orbit of $(z, w)$ under the moment map $\mu_{1}$ is the set

$$
\left\{\sum_{\left\{i ; z_{i} \neq 0\right\}} t_{i} \alpha_{i}-\sum_{\left\{i ; w_{i} \neq 0\right\}} s_{i} \alpha_{i}+c_{1}: t_{i}, s_{i}>0\right\} \subset \mathfrak{n}^{*} .
$$

The proof of this is essentially the same as in [Gu2;Appendix 1]. The moment map restricted to the orbit is given in our case by the Legendre transform of the function $F: \mathfrak{n}^{\mathbb{C}} \cap \mathbb{R}^{d} \rightarrow \mathbb{R}$ defined by

$$
F(y)=\frac{1}{4} \sum_{\left\{i ; z_{i} \neq 0\right\}} a_{i} e^{2 \alpha_{i} \cdot y}+\frac{1}{4} \sum_{\left\{i ; w_{i} \neq 0\right\}} b_{i} e^{-2 \alpha_{i} \cdot y}+c_{1} \cdot y,
$$


where $a_{i}, b_{i}$ are positive constants. This is a strictly convex function and all the arguments of Guillemin go through.

If $(z, w)$ is a point of $W$ then, from the proof of 3.1, we know that $\sum_{k=1}^{d}\left(z_{k} w_{k}+\lambda_{k}^{2}+\sqrt{-1} \lambda_{k}^{3}\right) e_{k}=\beta^{*}(b)$ for some $b \in \mathbb{C}^{n}$. If for all $k$ we have $\left\langle b, u_{k}\right\rangle \neq \lambda_{k}^{2}+\sqrt{-1} \lambda_{k}^{3}$, then $z_{k} w_{k}$ is nonzero for all $k$ and so the full group $\left(T^{d}\right)^{\mathbb{C}}$ acts freely at $(z, w)$. From (5.2), as the vectors $\alpha_{i}$ span $\mathfrak{n}^{*}$, the restriction of $\mu_{1}$ to $N^{\mathbb{C}}(z, w)$ is surjective.

On the other hand, if $\left\langle b, u_{k}\right\rangle=\lambda_{k}^{2}+\sqrt{-1} \lambda_{k}^{3}$ precisely when $k \in I$, where $I$ is some multi-index, then $z_{k} w_{k}=0$ if and only if $k \in I$. In particular the stabiliser group of $(z, w)$ for the action of $N^{\mathbb{C}}$ is a subgroup of $T_{I}^{\mathbb{C}}$. Since the flats $H_{k}^{2} \times H_{k}^{3}, k \in I$, now have nonempty intersection, the assumption of the theorem and the argument at the beginning of the proof of Theorem 3.2 imply that the vectors $u_{k}, k \in I$, are independent. Therefore the map $\beta$ sending $e_{i}$ to $u_{i}$ must be injective on $\mathbb{R}_{I}^{d}=\operatorname{Lie}\left(T_{I}\right)$. and, from (2.3),(2.4), we see that $\mathfrak{n} \cap \mathbb{R}_{I}^{d}=0$. The analogous statement for complex vector spaces is proved similarly, so the stabiliser for the $N^{\mathbb{C}}$ action is discrete. We also see that $\mathbb{R}^{d}=\left(\mathbb{R}_{I}^{d}\right)^{\perp}+\mathfrak{n}^{\perp}=\left(\mathbb{R}_{I^{c}}^{d}\right)+\mathfrak{n}^{\perp}$, and, since $i^{*}$ is just the orthogonal projection onto $\mathfrak{n} \equiv \mathfrak{n}^{*}$, it follows that $\mathfrak{n}^{*}$ is spanned by the set $\left\{\alpha_{i} ; i \notin\right.$ $I\}$. Therefore, from (5.2), $\mu_{1}$ is still surjective on $N^{\mathbb{C}}(z, w)$. This proves Theorem 5.1.

Example 5.2. Consider the hyperkähler quotient corresponding to Example 2.1 with $n=1$ (the Eguchi-Hanson space). The variety $W$ is described by the two equations $z_{1} w_{1}=b-\nu_{1}$ and $z_{2} w_{2}=-b-\nu_{2}$, where $\nu_{k}=\lambda_{k}^{2}+\sqrt{-1} \lambda_{k}^{3}$. The assumption of Theorem 5.1 is satisfied if $\nu_{1} \neq-\nu_{2}$. Eliminating $b$, we can view $W$ as the hypersurface in $\mathbb{C}^{4}$ with equation $z_{1} w_{1}+z_{2} w_{2}=\tau$, where $\tau \neq 0$. The ring of invariant polynomials for the action of $N^{\mathbb{C}} \cong \mathbb{C}^{*}$ is generated by $z_{1} w_{2}, z_{2} w_{1}, z_{1} w_{1}$. We find that $\left(M, J_{1}\right)$ is biholomorphic to the variety $x y=z(\tau-z)$ which can be viewed as a deformation of the Kleinian singularity $\mathbb{C}^{2} / \mathbb{Z}_{2}[\mathrm{Hi}]$.

\section{Topology of toric hyperkähler orbifolds.}

Our next task is to show how the vectors $u_{1}, \ldots, u_{d} \in \mathbb{R}^{n}$ and $\lambda_{1}, \ldots, \lambda_{d} \in$ $\mathbb{R}^{3}$ determine the topology of $M(\underline{u}, \underline{\lambda})$. First of all we have

Theorem 6.1. If $M(\underline{u}, \underline{\lambda})$ and $M\left(\underline{u}^{\prime}, \underline{\lambda}^{\prime}\right)$ are toric hyperkähler orbifolds, and $\underline{u}=\underline{u}^{\prime}$, then $M(\underline{u}, \underline{\lambda})$ is homeomorphic to $M\left(\underline{u}^{\prime}, \underline{\lambda}^{\prime}\right)$. 
In other words the homeomorphism type of $M$ depends only on the torus $N$ and not on the moment map (3.3). Before proving this let us establish a few related facts.

Proposition 6.2. Let $M(\underline{u}, \underline{\lambda})$ be a toric hyperkähler orbifold such that every $n+1$ hyperplanes $H_{k}^{1}$ have empty intersection. Then $M(\underline{u}, \underline{\lambda})$ is diffeomorphic to $M\left(\underline{u}, \underline{\lambda}^{\prime}\right)$, where $\lambda_{k}^{\prime}=\left(\lambda_{k}^{1}, 0,0\right)$ for each $k$.

Proof. Let us write $M\left(\underline{\lambda^{1}}, \underline{\lambda^{2}}, \underline{\lambda^{3}}\right)$ for $M(\underline{u}, \underline{\lambda})$. Applying Theorem 5.1 with respect to the complex structure $J_{3}$ shows that $M\left(\underline{\lambda^{1}}, \underline{\lambda^{2}}, \underline{\lambda^{3}}\right)$ is diffeomorphic to $M\left(\underline{\lambda^{1}}, \underline{\lambda^{2}}, 0\right)$. Applying it again, with respect to $J_{2}$, shows that $M\left(\underline{\lambda^{1}}, \underline{\lambda^{2}}, 0\right)$ is diffeomorphic to $M\left(\underline{\lambda^{1}}, 0,0\right)$.

If the condition of Theorem 3.2 or Theorem 3.3 is satisfied, then the hypothesis of 6.2 holds for a generic direction in $\mathbb{R}^{3}$. More precisely:

Lemma 6.3. Suppose that we are given vectors $u_{1}, \ldots, u_{d}$ generating $\mathbb{R}^{n}$ and elements $\lambda_{1}, \ldots, \lambda_{d}$ of $\mathbb{R}^{3}$ such that every $n+1$ flats $H_{k}$ defined by (3.4)-(3.5) have empty intersection. Then for a generic element $(a, b, c)$ of the 2 -sphere in $\mathbb{R}^{3}$, every $n+1$ of the hyperplanes $\left\{y \in \mathbb{R}^{n}:\left\langle y, u_{k}\right\rangle=\right.$ $\left.a \lambda_{k}^{1}+b \lambda_{k}^{2}+c \lambda_{k}^{3}\right\}$ have empty intersection.

Proof. If not, then there is a particular set of $n+1$ indices, say $1, \ldots, n+1$, such that the set $S$ of $(a, b, c)$ for which the corresponding $n+1$ hyperplanes intersect spans $\mathbb{R}^{3}$. Now for each $(a, b, c) \in S$ there exists $x_{a b c}$ such that $\left\langle x_{a b c}, u_{k}\right\rangle=a \lambda_{k}^{1}+b \lambda_{k}^{2}+c \lambda_{k}^{3}$ for $k=1, \ldots, n+1$. As $S$ spans $\mathbb{R}^{3}$, by taking linear combinations of various $x_{a b c}$ we can easily find a common point of the flats $H_{1}, \ldots, H_{n+1}$, contradicting the assumption of the lemma.

Lemma 6.4. Let $M(\underline{u}, \underline{\lambda})$ and $M\left(\underline{u}, \underline{\lambda}^{\prime}\right)$ be two toric hyperkähler orbifolds such that there is an element $A$ of $S O(3)$ with $A \lambda_{k}=\lambda_{k}^{\prime}$ for $k=1, \ldots, d$. Then $M(\underline{u}, \underline{\lambda})$ and $M\left(\underline{u}, \underline{\lambda}^{\prime}\right)$ are $T^{n}$-equivariantly diffeomorphic.

Proof. The right diagonal action on $\mathbb{H}^{d}$ of an element of $S p(1)$ covering $A$ induces a $T^{d}$-equivariant diffeomorphism of the two level sets.

Proof of Theorem 6.1. Because of Proposition 6.2 and Lemmas 6.3, 6.4 we can assume that all $\lambda_{k}$ and $\lambda_{k}^{\prime}$ lie on the $x_{1}$-axis. Let $U$ be the set of vectors 
$\lambda^{1}=\left(\lambda_{1}^{1}, \ldots, \lambda_{d}^{1}\right) \in \mathbb{R}^{d}$ such that $M(\underline{u}, \underline{\lambda})$ is an orbifold. The complement of $U$ is the set of $\lambda^{1}$ for which $n+1$ of the hyperplanes

$$
H_{k}^{1}=\left\{x \in \mathbb{R}^{n}:\left\langle x, u_{k}\right\rangle=\lambda_{k}^{1}\right\}
$$

have nonempty intersection. We shall first show that the topology of $M$ does not change as long as we stay within a connected component of $U$. If $\lambda^{1}$ and $\lambda^{1 \prime}$ lie in the same component of $U$, then there is a homeomorphism $h$ of $\mathbb{R}^{n}$ onto itself mapping each half-space $\left\{x \in \mathbb{R}^{n}:\left\langle x, u_{k}\right\rangle \leq \lambda_{k}^{1}\right\}$ onto the corresponding half-space $\left\{x \in \mathbb{R}^{n}:\left\langle x, u_{k}\right\rangle \leq \lambda_{k}^{1 \prime}\right\}$ and similarly for the opposite half-spaces. We consider now, as in [Go], the homeomorphism $\tau$ between $\mathbb{R}_{\geq 0} \times \mathbb{R}_{\geq 0}$ and $\mathbb{R} \times \mathbb{R}_{\geq 0}$ given by

$$
\tau(x, y)=\left(\frac{1}{2}\left(x^{2}-y^{2}\right), x y\right)
$$

which we extend diagonally to a homeomorphism, also denoted by $\tau$, between $\left(\mathbb{R}_{\geq 0} \times \mathbb{R}_{\geq 0}\right)^{d}$ and $\left(\mathbb{R} \times \mathbb{R}_{\geq 0}\right)^{d}$. Let $V(\underline{\lambda})$ be the subset of $\mathbb{R}^{d}$ consisting of vectors $p=\left(p_{1}, \ldots, p_{d}\right)$ such that there is an $a \in \mathbb{R}^{n}$ with $p_{k}=\left\langle a, u_{k}\right\rangle-\lambda_{k}^{1}, k=1, \ldots, d$. Since the vectors $u_{k}$ generate $\mathbb{R}^{n}$, the map $v: V(\underline{\lambda}) \rightarrow \mathbb{R}^{n}$ sending $p$ to $a$ is a homeomorphism. We define $V\left(\underline{\lambda}^{\prime}\right)$ and $v^{\prime}$ similarly . Let us extend $h, v, v^{\prime}$ to homeomorphisms $h: \mathbb{R}^{n} \times\left(\mathbb{R}_{\geq 0}\right)^{d} \rightarrow \mathbb{R}^{n} \times\left(\mathbb{R}_{\geq 0}\right)^{d}, v: V(\underline{\lambda}) \times\left(\mathbb{R}_{\geq 0}\right)^{d} \rightarrow \mathbb{R}^{n} \times\left(\mathbb{R}_{\geq 0}\right)^{d}$ and $v^{\prime}: V\left(\underline{\lambda}^{\prime}\right) \times\left(\mathbb{R}_{\geq 0}\right)^{d} \rightarrow \mathbb{R}^{n} \times\left(\mathbb{R}_{\geq 0}\right)^{d}$ by putting the identity map on the second factor. The composition $\Phi=\tau^{-1} \circ\left(v^{\prime}\right)^{-1} \circ h \circ v \circ \tau$ gives a homeomorphism between $\tau^{-1}\left(V(\underline{\lambda}) \times\left(\mathbb{R}_{\geq 0}\right)^{d}\right)$ and $\tau^{-1}\left(V\left(\underline{\lambda}^{\prime}\right) \times\left(\mathbb{R}_{\geq 0}\right)^{d}\right)$. Finally we define $\pi: \mathbb{C}^{d} \times \mathbb{C}^{d} \rightarrow\left(\mathbb{R}_{\geq 0} \times \mathbb{R}_{\geq 0}\right)^{d}$ by $\pi(z, w)=\left(\left|z_{1}\right|,\left|w_{1}\right|, \ldots,\left|z_{d}\right|,\left|w_{d}\right|\right)$. Let us write $\Phi \circ \pi=\left(\Phi_{1}^{1}, \Phi_{1}^{2}, \ldots, \Phi_{d}^{1}, \Phi_{d}^{2}\right)$. We can now define a $T^{d}$-equivariant homeomorphism $\Psi$ between the zero level sets for $\underline{\lambda}$ and $\underline{\lambda}^{\prime}$ by putting

$$
\begin{aligned}
& \Psi(z, w) \\
& \quad=\left(\Phi_{1}^{1}(z, w) \frac{z_{1}}{\left|z_{1}\right|}, \ldots, \Phi_{d}^{1}(z, w) \frac{z_{d}}{\left|z_{d}\right|}, \Phi_{1}^{2}(z, w) \frac{w_{1}}{\left|w_{1}\right|}, \ldots, \Phi_{d}^{2}(z, w) \frac{w_{d}}{\left|w_{d}\right|}\right) .
\end{aligned}
$$

This map induces a $T^{n}$-equivariant homeomorphism between $M(\underline{u}, \underline{\lambda})$ and $M\left(\underline{u}, \underline{\lambda}^{\prime}\right)$.

We have shown that as long as $\lambda^{1}$ does not pass through a critical value, i.e. a value for which $n+1$ hyperplanes (6.1) have nonempty intersection, then the topology of $M(\underline{u}, \underline{\lambda})$ does not change. We shall now show that it does not change even when we pass through a critical value. Let $\lambda^{1}=$ $\left(\lambda_{1}^{1}, \ldots, \lambda_{d}^{1}\right)$ be a critical value. We can assume that it is the hyperplanes 
$H_{1}^{1}, \ldots, H_{n+1}^{1}$ that have a nonempty intersection. We can also assume that $\left\{H_{1}^{1}, \ldots, H_{n+1}^{1}\right\}$ is a maximal set of hyperplanes with nonempty intersection, because the configurations with more than $n+1$ hyperplanes intersecting form a codimension 2 set in $\mathbb{R}^{d}$. Moreover, we can take $u_{2}, \ldots, u_{n+1}$ to be linearly independent, so that small perturbations of $\lambda_{1}$ will make $M(\underline{u}, \underline{\lambda})$ an orbifold.

Let $U_{-}$(resp. $\left.U_{+}\right)$denote the component of $U$ to which $\left(\lambda_{1}^{1}-\right.$ $\delta, \lambda_{2}^{1}, \ldots, \lambda_{d}^{1}$ ) belongs for a small positive (resp. negative) $\delta$. It will be enough to show that the topology of $M$ does not change as we pass from $U_{-}$to $U_{+}$. Let us consider an orbifold $M(\underline{u}, \underline{\lambda})$ where $\underline{\lambda}$ is obtained from $\left(\lambda_{1}, \ldots, \lambda_{d}\right)$ by replacing $\lambda_{1}=\left(\lambda_{1}^{1}, 0,0\right)$ with $\left(\lambda_{1}^{1}, \delta_{2}, \delta_{3}\right)$ for small $\delta_{2}, \delta_{3}$. Using Lemma 6.3 we can obtain a toric hyperkähler orbifold by projecting $\underline{\lambda}$ onto the subspace $\mathbb{R}(a, b, c) \otimes \mathbb{R}^{d}$ for generic small $b, c$ and $a$ close to 1 . Now we can use Lemma 6.4 to obtain an element $\lambda(b, c)$ of $\mathbb{R}^{d}=\mathbb{R}(1,0,0) \otimes \mathbb{R}^{d}$ such that the corresponding $M(\underline{u}, \underline{\lambda})$ is an orbifold. Moreover Proposition 6.2 and Lemma 6.4 show that the topology of this orbifold does not depend on $(b, c)$. However, by changing the signs of $b$ and $c$ we can guarantee that $\lambda(b, c)$ belongs to $U_{-}$for some choices of $(b, c)$, and belongs to $U_{+}$for other choices. This proves Theorem 6.1.

We shall now discuss the homotopy type of $M(\underline{u}, \underline{\lambda})$. Because of Theorem 6.1 we can assume that the vectors $\lambda_{k}$ are of the form $\left(\lambda_{k}^{1}, 0,0\right)$.

In what follows we shall use a similar argument to that of Goto [Go]. We shall consider the hyperplanes $H_{k}^{1}$ defined by (6.1). These hyperplanes divide $\mathbb{R}^{n}$ into a finite family of closed convex polyhedra, some unbounded. Let $\mathcal{A}$ be the polyhedral complex consisting of all faces of all dimensions of these polyhedra. We recall that a polyhedral complex is a family of polyhedra such that every face of a member of $\mathcal{A}$ is itself a member of $\mathcal{A}$ and the intersection of any two members of $\mathcal{A}$ is a face of each of them. We define the polyhedral (in fact polytopal) complex $\mathcal{C}$ to consist of all bounded polyhedra in $\mathcal{A}$. This complex is nonempty since, as the vectors $u_{k}$ generate $\mathbb{R}^{n}, \mathcal{C}$ must contain a vertex corresponding to the intersection of $n$ hyperplanes $H_{k}^{1}$. We index the elements of $\mathcal{C}$ by some set $I$ and denote the polyhedra in $\mathcal{C}$ by $\Delta_{s}, s \in I$. Finally, we denote by $|\mathcal{C}|$ the support $\bigcup_{s \in I} \Delta_{s}$ of the complex.

Recall that $\phi=\left(\phi_{1}, \phi_{2}, \phi_{3}\right): M \rightarrow \mathbb{R}^{n} \times \mathbb{R}^{n} \times \mathbb{R}^{n}$ is the moment map for the action of $T^{n}$ on $M$. We define subsets $X_{s}$ of $M$ by

$$
X_{s}=\phi^{-1}\left(\Delta_{s}, 0,0\right), \quad s \in I .
$$

The following result describes the topology of $M(\underline{u}, \underline{\lambda})$. 
Theorem 6.5. Let $M=M(\underline{u}, \underline{\lambda})$ be a toric hyperkähler orbifold, where $\lambda_{k}=\left(\lambda_{k}^{1}, 0,0\right)$ for each $k$. Then:

(i) $\bigcup_{s \in I} X_{s}=\phi^{-1}(|\mathcal{C}|, 0,0)$ is a $T^{n}$-equivariant deformation retract of $M$.

(ii) Each $X_{s}$ is a Kähler subvariety of $\left(M, J_{1}, \omega_{1}\right)$, isotropic with respect to the form $\omega_{2}+i \omega_{3}$ and invariant under the $T^{n}$-action.

(iii) Each $X_{s}$ is $T^{m}$-equivariantly isometric and biholomorphic to the toric variety determined by the polytope $\Delta_{s}$, where $T^{m}$ is the subtorus of $T^{n}$ acting effectively on $X_{s}$.

Proof. Once more we consider the homeomorphism $\tau$ between $\mathbb{R}_{\geq 0} \times \mathbb{R}_{\geq 0}$ and $\mathbb{R} \times \mathbb{R}_{\geq 0}$ defined by (6.2). Let $j_{t}$ be the deformation map of $\mathbb{R} \times \mathbb{R}_{\geq 0}$ defined by $j_{t}(u, v)=(u, t v)$. Then the composite map $\jmath_{t}=\tau^{-1} \circ j_{t} \circ \tau$ is a deformation of $\mathbb{R}_{\geq 0} \times \mathbb{R}_{\geq 0}$. Let us write $\jmath_{t}(x, y)=\left(\jmath_{t}^{1}(x, y), \jmath_{t}^{2}(x, y)\right)$. Now we define a deformation of $\mathbb{C}^{2}$ by the map $h:[0,1] \times \mathbb{C}^{2} \rightarrow \mathbb{C}^{2}$ where

$$
h(t, z, w)=\left(\jmath_{t}^{1}(|z|,|w|) \frac{z}{|z|}, \jmath_{t}^{2}(|z|,|w|) \frac{w}{|w|}\right)
$$

and extend this diagonally to a deformation of $\mathbb{C}^{d} \times \mathbb{C}^{d}$. We observe that $h$ is $T^{d}$-equivariant and the moment map (3.2) satisfies

$\mu_{1} \circ h_{t}(z, w)=\mu_{1}(z, w), \quad\left(\left(\mu_{2}+\sqrt{-1} \mu_{3}\right) \circ h_{t}\right)(z, w)=t\left(\mu_{2}+\sqrt{-1} \mu_{3}\right)(z, w)$

for any $t \in[0,1]$ (recall that we are setting $c_{2}=c_{3}=0$ ).

Therefore $h$ preserves the zero-set of (3.3). Since $h$ is $T^{d}$-equivariant, we obtain a $T^{n}$-equivariant deformation of $M$. Moreover $h_{0}(M)=\left(\phi_{2}+\right.$ $\left.\sqrt{-1} \phi_{3}\right)^{-1}(0)$, because of (3.6) and the fact that $b=\left(\phi_{2}+\sqrt{-1} \phi_{3}\right)(z, w)$.

We have now deformed $M$ to $\left(\phi_{2}+\sqrt{-1} \phi_{3}\right)^{-1}(0)$, which, by (3.6), corresponds to the quotient by $N$ of the set of $(z, w, a) \in \mathbb{C}^{d} \times \mathbb{C}^{d} \times \mathbb{R}^{n}$ such that

$$
z_{k} w_{k}=0, \quad \frac{1}{2}\left(\left|z_{k}\right|^{2}-\left|w_{k}\right|^{2}\right)+\lambda_{k}^{1}=\left\langle a, u_{k}\right\rangle, \quad(k=1, \ldots, d) .
$$

Let us recall once more that $a=\phi_{1}(z, w)$. We claim that there is a deformation map $p:[0,1] \times \mathbb{R}^{n} \rightarrow \mathbb{R}^{n}$, such that $p(1, a)=a$, the map $a \mapsto p(0, a)$ is a retraction onto $|\mathcal{C}|=\bigcup_{s \in I} \Delta_{s}$, and, if $a$ lies on a hyperplane $H_{k}^{1}$, then $p(t, a)$ lies on this hyperplane for all $t \in[0,1]$. To see this we observe that the complement of $|\mathcal{C}|=\bigcup_{s \in I} \Delta_{s}$ in $\mathbb{R}^{n}$ is a union of convex unbounded 
polyhedra $K_{i}$ with non-empty interior such that the intersection of any two of them will be a common face (of positive codimension) of each. Moreover each $K_{i}$ is line-free (if $K_{i}$ contains a line, spanned by a vector $v$, then $v$ is parallel to all hyperplanes $H_{k}^{1}$ hence orthogonal to all $u_{k}$, contradicting the assumption that the vectors $u_{k}$ span $\mathbb{R}^{n}$ ). Therefore we can think of each $K_{i}$ as a convex polytope $P$ whose unique face at infinity $F_{0}$ has been removed. We can find a deformation retraction of $K_{i}=P-F_{0}$ onto the part of the boundary consisting of bounded faces. Moreover we can assume that this deformation of $P-F_{0}$ is an extension of any given deformation of $\partial P-F_{0}$. Therefore, by doing it first on the intersections of $K_{i}$ 's and then extending to their interiors, we can define the desired deformation map $p$.

For $k=1, \ldots, d$, put $p_{t}^{k}(a)=2\left\langle p(t, a), u_{k}\right\rangle-2 \lambda_{k}^{1}$. We now define a $T^{d}$-equivariant deformation $f_{t}$ of the set given by (6.5):

$$
f_{t}\left(z_{k}, w_{k}\right)= \begin{cases}(0,0) & \text { if } \quad z_{k}=w_{k}=0 \\ \left(\frac{\sqrt{p_{t}^{k}(a)}}{\left|z_{k}\right|} z_{k}, 0\right) & \text { if } \quad z_{k} \neq 0 \\ \left(0, \frac{\sqrt{-p_{t}^{k}(a)}}{\left|w_{k}\right|} w_{k}\right) & \text { if } \quad w_{k} \neq 0\end{cases}
$$

and

$$
f_{t}(a)=p(t, a)
$$

Observe that $\phi_{1}\left(f_{t}(z, w)\right)=p(t, a)$. The deformation $f_{t}$ induces a $T^{n_{-}}$ equivariant deformation of $\left(\phi_{2}+\sqrt{-1} \phi_{3}\right)^{-1}(0)$ onto $\bigcup_{s \in I} X_{s}$, proving part (i).

For (ii)-(iii) we observe, as in [Go], that each $\mathcal{D}_{s}$ can be obtained as a Kähler quotient of a submanifold of $\mathbb{C}^{d} \times \mathbb{C}^{d}$ by the construction of section 2 for the polytope $\Delta_{s}$. These submanifolds are Kähler with respect to $\omega_{1}$ and isotropic with respect to $\omega_{2}+\sqrt{-1} \omega_{3}$, so all statements of (ii)-(iii) follow.

Recall that an arrangement of hyperplanes in $\mathbb{R}^{n}$ is simple if no $n+1$ of them intersect.

Corollary 6.6. If the arrangment of hyperplanes (6.1) is simple, then the homotopy type of the compact variety of Theorem 6.5(i) depends only on the vectors $u_{k}$.

There is a very simple formula relating the Betti numbers of a compact toric orbifold to the combinatorics of the corresponding convex polytope [Fu]. It turns out that a similar formula holds for toric hyperkähler orbifolds. 
Theorem 6.7. Let $M=M(\underline{u}, \underline{\lambda})$ be a toric hyperkähler orbifold of dimension $4 n$. Then $M$ is simply connected, $H^{j}(M, \mathbb{Q})=0$ if $j$ is odd or $j>2 n$, and the Betti numbers in the remaining even dimensions are given by

$$
b_{2 k}=\sum_{i=k}^{n}(-1)^{i-k}\left(\begin{array}{l}
i \\
k
\end{array}\right) d_{i}, \quad(k=1, \ldots, n)
$$

where $d_{i}$ denotes the number of $i$-dimensional elements of the complex $\mathcal{C}$. The Poincaré polynomial of $M$ is therefore

$$
\sum_{k=0}^{n} d_{k}\left(t^{2}-1\right)^{k} .
$$

In particular the Euler characteristic of $M$ is $d_{0}$, the number of vertices of $\mathcal{C}$.

Remark. For toric hyperkähler manifolds, $H^{*}(M, \mathbb{Z})$ has no torsion, so Theorem 6.7 tells us the cohomology over the integers.

We need first the following result.

Proposition 6.8. Let $\mathcal{C}$ be a polytopal complex determined by a simple arrangement of hyperplanes (6.1) and suppose that $|\mathcal{C}|$ is not contained in any hyperplane. Then every element of $\mathcal{C}$ which is maximal with respect to inclusion has dimension $n$.

Proof. Suppose, for a contradiction, that there is a maximal element $F$ of $\mathcal{C}$ with $\operatorname{dim} F=m \leq n-1$. We can suppose that $m \geq 1$. Let us choose one of the vertices of $F$ to be the origin, and the edges meeting at this vertex to be generated by an orthonormal basis $e_{1}, \ldots, e_{n}$ of $\mathbb{R}^{n}$. In other words we put $u_{i}=e_{i}$ and $\lambda_{i}^{1}=0$ for $i=1, \ldots, n$. We can further suppose that $F \subset \bigcap_{i=1}^{m}\left\{x \in \mathbb{R}^{n}:\left\langle x, e_{i}\right\rangle \geq 0\right\} \cap \bigcap_{i=m+1}^{n}\left\{x \in \mathbb{R}^{n}:\left\langle x, e_{i}\right\rangle=\right.$ $0\}$. The hyperplanes (6.1) divide $\mathbb{R}^{n}$ into finitely many closed convex $n$ dimensional polyhedra and, by assumption, $F$ is a face only of unbounded ones. Each of the polyhedra having $F$ for a face is determined by a function $\epsilon:\{m+1, \ldots, n\} \rightarrow\{-1,+1\}$. The polyhedron $P^{\epsilon}$ is then defined by having nonempty intersection with $\bigcap_{i=1}^{m}\left\{x:\left\langle x, e_{i}\right\rangle=0\right\} \cap \bigcap_{i=m+1}^{n}\{x$ : $\left.\left\langle x, e_{i}\right\rangle \cdot \epsilon(i)>0\right\}$. Since these are unbounded polyhedra, each of them contains a ray. In fact, for each function $\epsilon$ there is a $y^{\epsilon}$ with $\left\langle y^{\epsilon}, e_{i}\right\rangle \cdot \epsilon(i)>0$, $(i>m)$, and such that the ray $R_{x}^{\epsilon}=\left\{x+t y^{\epsilon}: t \geq 0\right\}$ is contained in $P^{\epsilon}$ for all $x \in F$. Note that $\left\langle y^{\epsilon}, e_{i}\right\rangle \geq 0$ for $i \leq m$. 
Suppose first that for all $i \leq m$, and for all $\epsilon$, we have $\left\langle y^{\epsilon}, e_{i}\right\rangle=0$. It is straightforward to show that if $k>n$ then $u_{k} \in \operatorname{Span}\left\{e_{1}, \ldots, e_{m}\right\}$ (otherwise one of the rays meets $H_{k}^{1}$, giving a contradiction). So $|\mathcal{C}| \subset$ $\operatorname{Span}\left\{e_{1}, \ldots, e_{m}\right\}$, which contradicts the assumption of the proposition.

Let us assume, then, that there exist $i_{0} \leq m$, and $\epsilon$, such that $\left\langle y^{\epsilon}, e_{i_{0}}\right\rangle>$ 0 . If we remove the hyperplanes $H_{m+1}^{1}, \ldots, H_{n}^{1}$, the remaining hyperplanes still divide $\mathbb{R}^{n}$ into convex $n$-dimensional polyhedra $P_{j}$. It follows that a neighborhood of any interior point of $F$ belongs now to a single $P_{j_{0}}$ and, consequently, the rays $R_{x}^{\epsilon},(x \in F)$, are all contained in $P_{j_{0}}$. Since $\epsilon$ ranges over all sign combinations of the last $n-m$ coordinates, we can find $z$ in the interior of the convex hull of the $y^{\epsilon}$ such that $\left\langle z, e_{i}\right\rangle=0$ for $i>m$. Observe that $\left\langle z, e_{i}\right\rangle$ is nonnegative for $i \leq m$, and is positive for $i=i_{0}$.

Since $P_{j_{0}}$ is convex, we see that for any $x$ in the interior of $F$, the ray $R=\{x+t z: t>0\}$ is contained in the interior of $P_{j_{0}}$. As $\left\langle z, e_{i}\right\rangle=0$ for $i>m$, this ray must meet an $(m-1)$-dimensional face of $F$, and so meets a hyperplane $H_{k}^{1}$ with $k>n$, contradicting the fact that $R$ lies in the interior of $P_{j_{0}}$.

Proof of Theorem 6.7. We observe first that both sides of (6.6) depend only on the vectors $u_{k}$. Indeed, Theorem 6.1 shows that it is so for the Betti numbers. On the other hand, moving one of the hyperplanes (6.1) in the direction of its orthogonal does not change the number of $k$-dimensional faces of $|\mathcal{C}|$, as long as the initial and final hyperplane arrangments are simple. Also, Theorem 6.1 shows that the other statements of Theorem 6.7 depend only on the $u_{k}$.

We proceed now by induction on $n$. The result is easily verified if $n=1$. Suppose that $n>1$, and that the theorem holds for $k<n$. In dimension $n$ we proceed by induction on the number $d$ of hyperplanes. Our statements hold for $n$ hyperplanes with a nonempty intersection. Suppose that they hold for $q$ hyperplanes in $\mathbb{R}^{n}$ whenever $n \leq q \leq d-1$. Now consider a toric hyperkähler orbifold $M(\underline{u}, \underline{\lambda})$ corresponding to hyperplanes $H_{1}^{1}, \ldots, H_{d}^{1}$. Because of Proposition 6.8 we can suppose that $\operatorname{dim}|\mathcal{C}|=n$. By the remark above we can move the hyperplane $H_{d}^{1}$ until all of $|\mathcal{C}|$ lies to one side of $H_{d}^{1}$, say $|\mathcal{C}| \subset\left\{x:\left\langle x, u_{d}\right\rangle \geq \lambda_{d}^{1}\right\}$. The intersections of $H_{d}^{1}$ with the $H_{k}^{1}, k<d$, determine a simple arrangement of hyperplanes in $H_{d}^{1} \cong \mathbb{R}^{n-1}$ which gives a toric hyperkähler orbifold $Y$ of real dimension $4 n-4$. Let us denote its polytopal complex by $\mathcal{E}$. On the other hand the hyperplanes $H_{1}^{1}, \ldots, H_{d-1}^{1}$ also determine a toric hyperkähler orbifold $W$ with a polytopal complex $\mathcal{G}$. By the inductive hypothesis, the theorem holds for $Y$ and $W$. We observe 
that, as every maximal element of $\mathcal{C}$ has dimension $n$, every $i$-dimensional element of $\mathcal{E}$ is a face of an $(i+1)$-dimensional element of $\mathcal{C}$. This implies that, if $e_{k}, g_{k}$ denote the number of $k$-dimensional faces of $\mathcal{E}, \mathcal{G}$ respectively, then $d_{0}=g_{0}+e_{0}$ and $d_{k}=g_{k}+e_{k}+e_{k-1}$ for $k$ positive.

Let us now pick a suitably small, positive, $\delta$, and consider the neigbourhoods of $|\mathcal{E}|$ and $|\mathcal{G}|$ in $|\mathcal{C}|$ defined by $U_{1}=|\mathcal{C}| \cap\left\{x \in \mathbb{R}^{n}:\left\langle x, u_{d}\right\rangle\left\langle\lambda_{d}^{1}+2 \delta\right\}\right.$ and $U_{2}=|\mathcal{C}| \cap\left\{x \in \mathbb{R}^{n}:\left\langle x, u_{d}\right\rangle>\lambda_{d}^{1}+\delta\right\}$. We also consider the deformation retract $X$ of $M$ given by Theorem 6.5(i). We have $X=V_{1} \cup V_{2}$ where $V_{1}=\phi^{-1}\left(U_{1}, 0,0\right)$ and $V_{2}=\phi^{-1}\left(U_{2}, 0,0\right)$. Now, by the argument used in the proof of $6.5, V_{1}$ can be deformed onto the deformation retract of $Y$ defined by Theorem 6.5(i) and so $V_{1}$ is homotopy equivalent to $Y$. Similarly $V_{2}$ is homotopy equivalent to $W$. Moreover, we see using 6.8 that $V_{1} \cap V_{2}$ is homotopy equivalent to an $S^{1}$-bundle $E$ over $Y$. We deduce that $M$ is simply-connected. Note that $E \rightarrow Y$ is an orientable bundle, as $Y$ is simply-connected.

We now consider the Mayer-Vietoris sequence for $V_{1}, V_{2}$ and the Gysin sequence for $E \rightarrow Y$. The cohomology here is rational, but it can be taken integer if $M$ is a manifold. By the inductive hypothesis, the odd Betti numbers of $Y$ and $W$ vanish, so the Mayer-Vietoris and the Gysin sequences split off at each even level as

$$
\begin{aligned}
& 0 \rightarrow H^{2 k-1}(E) \rightarrow H^{2 k}(M) \rightarrow H^{2 k}(Y) \oplus H^{2 k}(W) \rightarrow \\
& \rightarrow H^{2 k}(E) \rightarrow H^{2 k+1}(M) \rightarrow 0, \\
& 0 \rightarrow H^{2 k-1}(E) \rightarrow H^{2 k-2}(Y) \rightarrow H^{2 k}(Y) \rightarrow H^{2 k}(E) \rightarrow 0 .
\end{aligned}
$$

The Gysin sequence implies that the map $H^{2 k}(Y) \rightarrow H^{2 k}(E)$ is onto, so the odd cohomology of $M$ vanishes. Comparing the two short sequences, we find that the even Betti numbers satisfy the relation $b_{2 k}(M)=b_{2 k}(W)+b_{2 k-2}(Y)$ for $k>0$ and $b_{0}(M)=b_{0}(W)$.

The result now easily follows from these relations, together with the inductive hypothesis, the above formulae relating $d_{k}, e_{k}, g_{k}$, and standard identities for binomial coefficients.

Example 6.9. Consider $M(\underline{u}, \underline{\lambda})$ where $u_{k}, \lambda_{k}$ are as in Example 2.2. The polytopes $\Delta_{s}$ of Theorem 6.5 are just two intervals with a common point, so the deformation retract of $M(\underline{u}, \underline{\lambda})$ given by this theorem is the union of two copies of $\mathbb{C} P^{1}$ intersecting at a point. This retract is the exceptional divisor of the resolution of $\mathbb{C}^{2} / \mathbb{Z}_{3}$. 
Example 6.10. Suppose $u_{k}, \lambda_{k}$ are as in Example 2.3, with $a=1$. For a suitable choice of $\lambda_{3}$, we see that $|\mathcal{C}|$ is the union of a trapezoid and an isosceles right triangle intersecting along a line segment (see Fig. 5). The deformation retract $X$ of $M$, given by Theorem 6.5 , is the union of $\mathbb{C P}^{2}$ and the blowup of $\mathbb{C P}^{2}$, intersecting along the exceptional divisor. We calculate, using (6.6), that $b_{2}=b_{4}=2$. If we decrease $\lambda_{3}$, then $|\mathcal{C}|$ becomes the union of two isosceles right triangles meeting in a point (Fig. 6). The deformation retract $X^{\prime}$ of $M$ is the union of two copies of $\mathbb{C} P^{2}$ intersecting at a point. Corollary 6.6 implies that $X$ and $X^{\prime}$ are homotopy equivalent.

Proposition 6.8 implies that the deformation retract of $M$ is always a pseudomanifold.

\section{Toric hyperkähler manifolds from polytopes.}

In this section we shall discuss the toric hyperkähler manifolds corresponding to a convex polytope $\Delta$ in $\mathbb{R}^{n}$. That is, we shall consider $M(\underline{u}, \underline{\lambda})$ where $\underline{u}=\left(u_{1}, \ldots, u_{d}\right), \underline{\lambda}=\left(\lambda_{1}, \ldots, \lambda_{d}\right), \lambda_{k}=\left(\lambda_{k}^{1}, 0,0\right)$ and $\Delta$ is the intersection of half-spaces

$$
\left\langle x, u_{k}\right\rangle \geq \lambda_{k}^{1}, \quad(k=1, \ldots, d),
$$

as in $\S 2$. We shall always assume that $\Delta$ is simple, that is, there are precisely $n$ edges meeting at each vertex of $\Delta$. In this situation we shall write $M_{\Delta}$ for $M(\underline{u}, \underline{\lambda})$. It is useful to observe that with this choice of $\lambda_{k}$, a collection of flats $H_{k}$ intersect if and only if the corresponding collection of hyperplanes $H_{k}^{1}$ intersect.

We shall be particularly interested in the relation between $M_{\Delta}$ and the Kähler toric variety $X_{\Delta}$ obtained by the construction of section 2. First of all we shall show that the cotangent bundle of a toric manifold always carries a hyperkähler metric (usually incomplete).

Theorem 7.1. Let $X_{\Delta}$ be a smooth compact toric variety corresponding to a Delzant polytope $\Delta$. Then $T^{*} X_{\Delta}$ with its natural complex-symplectic structure is $T^{n}$-equivariantly isomorphic to an open subset $U_{\Delta}$ of the (usually singular) space $\left(M_{\Delta}, J_{1}, \omega_{2}+\sqrt{-1} \omega_{3}\right)$. If we identify $U_{\Delta}$ with $T^{*} X_{\Delta}$, the hyperkähler metric of $M_{\Delta}$ restricted to the zero section of $T^{*} X_{\Delta}$ is the Kähler metric on $X_{\Delta}$ determined by $\Delta$.

Proof. Consider the open subset $Y=\mathbb{C}_{\Delta}^{d} \times \mathbb{C}^{d}$ of $\mathbb{H}^{d} \simeq \mathbb{C}^{d} \times \mathbb{C}^{d}$, where $\mathbb{C}_{\Delta}^{d}$ is given by (2.10). Now $Y$ is a hyperkähler $T^{d}$-invariant submanifold of $\mathbb{H}^{d}$ so 
in particular is $N$-invariant, where $N$ denotes the torus of (2.5). Moreover the action of $N$ on $Y$ is free, because it is free on $\mathbb{C}_{\Delta}^{d}$. Therefore we can perform the hyperkähler quotient construction on $Y$ and obtain a smooth manifold $U_{\Delta}$ which is an open subset of $M_{\Delta}$. Note that $U_{\Delta}$ is preserved by the $T^{n}$ action on $M_{\Delta}$.

We want to identify $U_{\Delta}$, the hyperkähler quotient of $Y$ by $N$, with the complex-symplectic quotient of $Y$ by $N^{\mathbb{C}}$ (with respect to the complex structure $J_{1}$ ). For this we have to show that every $N^{\mathbb{C}}$ orbit in the intersection of $Y$ with the zero-set of $(3.3 \mathrm{~b})$ (where $c_{2}=c_{3}=0$ ) meets the zero-set of (3.3a). Let $(z, w)$ be in the zero-set of $(3.3 \mathrm{~b})$, where $z \in \mathbb{C}_{\Delta}^{d}$. From the proof of Theorem 5.1 we know that the image of the $N^{\mathbb{C}}$-orbit of $(z, w)$ under (3.3a) is $S=\left\{\sum_{\left\{i ; z_{i} \neq 0\right\}} t_{i} \alpha_{i}-\sum_{\left\{i ; w_{i} \neq 0\right\}} s_{i} \alpha_{i}+c_{1}: t_{i}, s_{i}>0\right\}$. We also know [Gu2] that the image under (2.6) is the set $\left\{\sum_{\left\{i ; z_{i} \neq 0\right\}} t_{i} \alpha_{i}+c_{1}: t_{i}>0\right\}$ and that for $z \in \mathbb{C}_{\Delta}^{d}$ this set is open. However, since $z \in \mathbb{C}_{\Delta}^{d}$, this last set contains 0 , so $S$ contains 0 .

We have shown that $\left(U_{\Delta}, J_{1}\right)$ is the complex-symplectic quotient of $Y$ by $N^{\mathbb{C}}$, and so is $\left\{(z, w) \in \mathbb{C}^{2 d}: \sum_{k=1}^{d}\left(z_{k} w_{k}\right) \alpha_{k}=0, z \in \mathbb{C}_{\Delta}^{d}\right\} / N^{\mathbb{C}}$. The equation in $z, w$ simply says that the vector $w \in T_{z}^{*} \mathbb{C}_{\Delta}^{d}$ annihilates the vertical tangent vectors of the projection $\mathbb{C}_{\Delta}^{d} \rightarrow \mathbb{C}_{\Delta}^{d} / N^{\mathbb{C}}=X_{\Delta}$. This shows that $\left(U_{\Delta}, J_{1}\right)$ is biholomorphic to $T^{*} X_{\Delta}$. It is also clear that the symplectic forms are the same, since the form $\omega_{2}+\sqrt{-1} \omega_{3}$ on $T^{*} \mathbb{C}_{\Delta}^{d}$ is just $\sum d z_{k} \wedge d w_{k}$. The statement about the metrics follows as in (iii) of Theorem 6.5.

The metric on $T^{*} X_{\Delta}$ is complete precisely when $U_{\Delta}=M_{\Delta}$. However our next result shows that this occurs only when $X_{\Delta}$ is the product of projective spaces.

Theorem 7.2. Let $X_{\Delta}$ be a smooth compact toric variety as in Theorem 7.1. Then the following conditions are equivalent:

(i) $U_{\Delta}=M_{\Delta}=T^{*} X_{\Delta}$,

(ii) if some collection of hyperplanes containing $(n-1)$-dimensional faces of $\Delta$ do not meet in $\Delta$, then they do not meet outside $\Delta$,

(iii) $X_{\Delta}$ is the product of projective spaces.

Proof. Observe that (ii) is equivalent to requiring that all vertices (intersections of $n$ hyperplanes) lie in $\Delta$. It is also clear from Theorem 6.5 or the 
remark after Theorem 3.1 that (ii) is necessary for (i). Let us show that it is sufficient.

Without loss of generality we can assume that $\Delta$ contains 0 in its interior, so all the $\lambda_{k}^{1}$ must be negative. Now, $\mu_{1}^{-1}(0)$ can be written as the set of $(z, w)$ satisfying

$$
\frac{1}{2} \sum_{k=1}^{d}\left|z_{k}\right|^{2} \alpha_{k}=\sum_{k=1}^{d}\left(\frac{1}{2}\left|w_{k}\right|^{2}-\lambda_{k}^{1}\right) \alpha_{k} .
$$

It follows that if $(z, w) \in \mu_{1}^{-1}(0)$, then $z$ lies in $\left(\mu_{1}^{\prime}\right)^{-1}(0)$ where $\mu_{1}^{\prime}$ is the moment map (2.6) with a different choice of $c$. Hence $z$ belongs to $\mathbb{C}_{\Delta^{\prime}}^{d}$ where $\Delta^{\prime}$ is the intersection of half-spaces (7.1) with $\lambda_{k}^{1}$ of possibly larger absolute value (note that (ii) implies that $\Delta^{\prime}$ is Delzant). Condition (ii) also shows that in fact $z \in \mathbb{C}_{\Delta}^{d}$. Hence the hyperkähler quotient of $\mathbb{H}^{d}$ by $N$ is the same as the hyperkähler quotient of $Y=\mathbb{C}_{\Delta}^{d} \times \mathbb{C}^{d}$ by $N$. The proof of Theorem 7.1 now shows that $U_{\Delta}=M_{\Delta}$.

The implication (iii) $\Rightarrow$ (ii) is obvious. Let us now show the converse. As usual, we denote by $u_{k_{i}}$ the vectors defining $\Delta$. We consider the fan $\mathcal{F}$ corresponding to the polytope $\Delta$ and defined at the end of section 2 . Condition (ii) implies that for any independent set of vectors $\left\{u_{k_{1}}, \ldots, u_{k_{s}}\right\}$ the cone $\left\{\sum t_{i} u_{k_{i}}: t_{i} \geq 0\right\}$ belongs to $\mathcal{F}$. Indeed, since the vectors are independent, the hyperplanes orthogonal to them must intersect, so by (ii) they intersect in $\Delta$.

From this two facts follow: 1) any vector in $\mathbb{R}^{n}$ can be written uniquely as $\sum t_{i} u_{k_{i}}$ with $t_{i}>0$ and $u_{k_{1}}, \ldots, u_{k_{s}}$ linearly independent; 2) if $\Delta^{\prime}$ is another Delzant polytope, then there are no nontrivial equivariant birational morphisms $X_{\Delta} \rightarrow X_{\Delta^{\prime}}$. For 1) notice that if a vector could be written thus in two ways, then then the cones spanned by the two sets of $u_{k_{i}}$ would intersect in their interior, contradicting the definition of the fan. For 2) we first recall [Od] that such a morphism corresponds to removing a number of $(n-1)$-dimensional walls in cones of the fan $\mathcal{F}$ of $X_{\Delta}$ to obtain the fan $\mathcal{F}^{\prime}$ of $X_{\Delta^{\prime}}$. Consider an $n$-dimensional cone $\sigma$ in $\mathcal{F}^{\prime}$ that is not in $\mathcal{F}$. If $\sigma$ is a cone over a simplex, then the vectors generating $\sigma$ are linearly independent and we get a contradiction as $\sigma \notin \mathcal{F}$. If $\sigma$ has more than $n$ generating vectors, then taking two independent $n$-element sets such that the cones spanned by them have $n$-dimensional intersection we obtain a contradiction with the fact that the intersection of two cones in $\mathcal{F}$ is a face of each of them.

We appeal now to Reid's version [Re] of Mori's theory for projective toric varieties (see also the exposition in [Od]). We can conclude from fact 2) above, and Corollary 2.28(1) and Theorem 2.27(2) in [Od], that $\mathbb{R}^{n}=\sum V_{i}$ 
where each $V_{i}$ is a vector space of positive dimension and each 1-dimensional cone of $\mathcal{F}$ lies in some $V_{i}$. Moreover, each $V_{i}$ is spanned by the cones it contains. (In Oda's terminology, the $V_{i}$ are the spaces $\pi_{+}(R)$ where $R$ ranges over the extremal rays of $N E\left(X_{\Delta}\right)$ ). We denote by $\mathcal{F}_{i}$ the restriction of $\mathcal{F}$ to $V_{i}$, that is, the cones of $\mathcal{F}_{i}$ are precisely the cones of $\mathcal{F}$ contained in $V_{i}$. Now Corollary 2.6 of [Re] shows that each $\mathcal{F}_{i}$ is a fan of a projective space of an appropriate dimension. It remains to show that the sum $\sum V_{i}$ is direct. Suppose that the sum $V_{1}+\ldots+V_{s}$ is direct and that $V_{s+1}$ intersects $\bigoplus_{1}^{s} V_{i}$ nontrivially. If $v$ lies in the intersection, then, because of the definition of the spaces $V_{i}$, it can be written as $\sum t_{i} u_{k_{i}}$ with $t_{i}>0, u_{k_{i}} \in \bigoplus_{1}^{s} V_{i}$ and also as $\sum s_{j} u_{l_{j}}$ with $s_{j}>0, u_{l_{j}} \in V_{s+1}$, where the $u_{k_{i}}$ and the $u_{l_{j}}$ are linearly independent. By fact 1) the two sets $\left\{u_{k_{i}}\right\}$ and $\left\{u_{l_{j}}\right\}$ are equal and the vectors $u_{k_{i}}$ must belong to both $\bigoplus_{1}^{s} V_{i}$ and to $V_{s+1}$. The vector $-u_{k_{1}}$ also belongs to both $\bigoplus_{1}^{s} V_{i}$ and to $V_{s+1}$. Moreover, since the fan $\mathcal{F}_{s+1}$ is the fan of a projective space, $-u_{k_{1}}$ belongs to the open cone in $\mathcal{F}_{s+1}$ generated by all 1-dimensional cones of $\mathcal{F}_{s+1}$ except $u_{k_{1}}$ and so it can be written as their combination with all coefficients positive. Repeating the previous argument with $v=-u_{k_{1}}$ shows that all 1-dimensional cones of $\mathcal{F}_{s+1}$ belong to $\bigoplus_{1}^{s} V_{i}$ and so $V_{s+1} \subset \bigoplus_{1}^{s} V_{i}$. In fact we have shown that any 1-dimensional cone of $\mathcal{F}_{s+1}$ is a 1-dimensional cone of some $\mathcal{F}_{i}, i \leq s$. However, each of these fans is the fan of a projective space, and the only way that all generators of a fan of a projective space can lie among generators of fans of other projective spaces lying in a direct sum of the relevant vector spaces is when $\mathcal{F}_{s+1}$ is equal to $\mathcal{F}_{i}$, for some $i \leq s$. Such a repetition does not alter the conclusion that $\mathcal{F}$ is the fan of a product of projective spaces.

We can also ask when $M_{\Delta}$ is smooth. This is equivalent to asking whether the hyperkähler metric on $T^{*} X_{\Delta}$ can be smoothly completed. Delzant's work shows that the toric variety $X_{\Delta}$ obtained by the construction of section 2 is smooth if and only if whenever $n$ of the defining hyperplanes meet at a vertex of the simple polytope $\Delta$, the corresponding vectors $u_{i}$ form a $\mathbb{Z}$-basis of $\mathbb{Z}^{n}$. This condition is not, however, sufficient for $M_{\Delta}$ to be smooth. Indeed, Theorem 3.2 requires that the Delzant condition holds at any intersection of $n$ hyperplanes even if the intersection is outside $\Delta$. In particular each of the varieties $X_{s}$ of Theorem 6.5 must be smooth.

Proposition 7.3. Let $X$ be a smooth projective toric variety of complex dimension $n$. Then the following statements are equivalent:

(i) $X$ carries a $T^{n}$-invariant Kähler metric such that, if $\Delta$ denotes the corresponding Delzant polytope, then $M_{\Delta}$ is smooth, 
(ii) every set of $n$ independent generators $u_{i}$ of the fan of $X$ is a $\mathbb{Z}$-basis of $\mathbb{Z}^{n}$.

Proof. The above discussion shows that (i) implies (ii). As $X$ is projective and toric it can be embedded equivariantly in projective space so admits a $T^{n}$-invariant Kähler metric, so can be obtained from the Delzant construction. As in Remark 3.4, by adjusting $\lambda_{i}$ we can choose an invariant Kähler metric on $X$ so that no $n+1$ flats intersect. Condition (ii), together with the argument at the beginning of 3.2, now shows that the condition of 3.2 holds, so $M_{\Delta}$ is smooth.

Remark 7.4. The argument of 3.4 shows that every smooth projective toric variety carries a $T^{n}$-invariant Kähler metric such that $M_{\Delta}$ is a hyperkähler completion of $T^{*} X_{\Delta}$ with at worst abelian quotient singularities.

Condition (ii) of Proposition 7.3 is rather restrictive. Let us choose an $n$-dimensional cone of $\mathcal{F}$, which we can take to be generated by vectors $e_{i}$, $(i=1, \ldots, n)$. Then any other generator $u_{i}$ of $\mathcal{F}$ must have coordinates in $\{-1,0,1\}$. In particular the number of 1 -dimensional cones of $\mathcal{F}$ is bounded by $3^{n}-2$ (we exclude the zero vector and $e_{1}+\ldots+e_{n}$ ) and so there only finitely many such varieties in each dimension. Proposition 7.3 can be used to show the following results.

Proposition 7.5. Let $X$ be a smooth compact toric variety of complex dimension 2 satisfying assumption (ii) of Proposition 7.3. Then $X$ is either $\mathbb{C} P^{1} \times \mathbb{C} P^{1}$ or the equivariant blow-up of $\mathbb{C} P^{2}$ at $k$ points, where $0 \leq k \leq 3$.

Proposition 7.6. Let $X_{\Delta}=\prod X_{i}$, where each $X_{i}$ is the equivariant blowup of $\mathbb{C} P^{n_{i}}$ at $k_{i}$ points, and $0 \leq k_{i} \leq n_{i}+1$. Then $M_{\Delta}$ is smooth.

\section{Kähler potentials.}

Guillemin has derived a formula for the Kähler form of a toric variety in terms of the associated polytope [Gu1]. We shall now find an expression in terms of $\underline{u}, \underline{\lambda}$ for the Kähler form, say $\omega_{1}$, on the hyperkähler manifold $M(\underline{u}, \underline{\lambda})$.

The Kähler form $\omega$ (and so the metric) of a Kähler manifold $X$ is locally determined by a Kähler potential, a real-valued function $K$ locally defined on $X$ such that $2 \sqrt{-1} \partial \bar{\partial} K=\omega$. In general, finding the Kähler potential 
of a hyperkähler manifold is a complicated problem. It is much simpler, however, when the $4 n$-dimensional hyperkähler manifold $M$ admits a free Hamiltonian action of an $n$-dimensional abelian group $G$ preserving the hyperkähler structure. Then $M$ described as a principal $G$-bundle over an open subset of $\mathbb{R}^{n} \otimes \mathbb{R}^{3}$ where is the projection is just the moment map $\phi=\left(\phi_{1}, \phi_{2}, \phi_{3}\right): M \rightarrow \mathbb{R}^{n} \otimes \mathbb{R}^{3}$. Since the group action preserves the hyperkähler structure, the Kähler potential with respect to any complex structure does not depend on the fiber coordinate. It is convenient to introduce the map $\pi=\left(2 \phi_{1}, \phi_{2}+\sqrt{-1} \phi_{3}\right): M \rightarrow \mathbb{R}^{n} \times \mathbb{C}^{n}$.

Theorem 8.1 [HKLR]. In the above situation the Kähler potential for the form $\omega_{1}$ on $M$ is

$$
K=\pi^{*}\left(F-\sum_{i=1}^{n} s_{i} \frac{\partial F}{\partial s_{i}}\right),
$$

where $F=F\left(s_{i}, v_{i}, \bar{v}_{i}\right)$ is a real-valued function on $\mathbb{R}^{n} \times \mathbb{C}^{n}$ satisfying the linear equations $F_{s_{i} s_{j}}+F_{v_{i} \bar{v}_{j}}=0,(1 \leq i, j \leq n)$.

Our manifolds fall into this class of examples with $G=T^{n}$, provided we restrict to an open dense subset.

Example 8.2. Consider the open subset of $\mathbb{H}^{d}$ on which the diagonal action of the torus $T^{d}$ is free. We have [HKLR]

$$
F(s, v, \bar{v})=\frac{1}{4} \sum_{i=1}^{d}\left(r_{i}-s_{i} \ln \left(s_{i}+r_{i}\right)\right),
$$

where $r_{i}^{2}=s_{i}^{2}+4 v_{i} \bar{v}_{i}$. Here $v_{i}, s_{i}$ are related to our standard coordinates $z_{i}, w_{i}$ by $v_{i}=z_{i} w_{i}$ and $s_{i}=\left|z_{i}\right|^{2}-\left|w_{i}\right|^{2}$. The Kähler potential is given by $K=\frac{1}{4} \sum r_{i}$.

We now want to calculate the Kähler potential for the form $\omega_{1}$ on our hyperkähler quotient $M=M(\underline{u}, \underline{\lambda})$ (or more precisely on the open dense subset where $T^{n}$ acts freely). Our metric was given as the hyperkähler quotient of the metric of Example 8.2 by some subtorus of $T^{d}$. In the coordinates $s_{i}, v_{i}$, the equations definining the zero-set of the moment map (3.3) become linear:

$$
\sum_{k=1}^{d}\left(s_{k}+2 \lambda_{k}^{1}\right) \alpha_{k}=0
$$




$$
\sum_{k=1}^{d}\left(v_{k}+\lambda_{k}^{2}+\sqrt{-1} \lambda_{k}^{3}\right) \alpha_{k}=0 .
$$

The function $F$ given by (8.2) restricts to the flats defined by(8.3), and gives the Kähler potential on $M$ via formula (8.1) [HKLR, section 2(C)]. Therefore all that remains to be done is to express this restricted function in terms of the coordinates $(a, b)=\left(\phi_{1}(m),\left(\phi_{2}+\sqrt{-1} \phi_{3}\right)(m)\right)$, where $\left(\phi_{1}, \phi_{2}, \phi_{3}\right)$ is the hyperkähler moment map for the action of $T^{n}$ on $M$.

Let $m \in M$, and suppose that the image of $m$ in $\mathbb{R}^{d} \times \mathbb{C}^{d}$ is a point $(s, v)$ satisfying (8.3). Using (3.6) we obtain:

$$
s_{k}=2\left\langle a, u_{k}\right\rangle-2 \lambda_{k}^{1}, \quad v_{k}=\left\langle b, u_{k}\right\rangle-\lambda_{k}^{2}-\sqrt{-1} \lambda_{k}^{3}
$$

and so we have the function $F$ for $\left(M, \omega_{1}\right)$. We calculate the Kähler potential according to (8.1) and obtain

$$
\begin{aligned}
F-\sum_{i=1}^{n} a_{i} \frac{\partial F}{\partial a_{i}} & =F-\sum_{i=1}^{n} \sum_{k=1}^{d} a_{i} \frac{\partial F}{\partial s_{k}} \frac{\partial s_{k}}{\partial a_{i}}=F-2 \sum_{i=1}^{n} \sum_{k=1}^{d} a_{i} \frac{\partial F}{\partial s_{k}}\left(u_{k}\right)_{i} \\
& =F-\sum_{k=1}^{d}\left(s_{k}+2 \lambda_{k}^{1}\right) \frac{\partial F}{\partial s_{k}}=\frac{1}{4} \sum_{k=1}^{d}\left(r_{k}+2 \lambda_{k}^{1} \ln \left(s_{k}+r_{k}\right)\right)
\end{aligned}
$$

where at the last step we use the equation

$$
\frac{\partial F}{\partial s_{k}}=\frac{1}{4} \sum_{k=1}^{d}\left(\frac{s_{k}}{r_{k}}-\ln \left(s_{k}+r_{k}\right)-\frac{s_{k}}{s_{k}+r_{k}}\left(1+\frac{s_{k}}{r_{k}}\right)\right)=-\frac{1}{4} \sum_{k=1}^{d} \ln \left(s_{k}+r_{k}\right) .
$$

This gives the next theorem, in which $\pi: M \rightarrow \mathbb{R}^{n} \times \mathbb{C}^{n}$ is the projection defined above, and $\partial_{1}$ is the Dolbeault operator corresponding to the complex structure $J_{1}$.

Theorem 8.3. On the open dense subset where the action of $T^{n}$ is free, the Kähler form $\omega_{1}$ on the toric hyperkähler manifold $M=M(\underline{u}, \underline{\lambda})$ is given by:

$$
\omega_{1}=\frac{\sqrt{-1}}{2} \partial_{1} \bar{\partial}_{1} \pi^{*}\left(\sum_{k=1}^{d}\left(r_{k}+2 \lambda_{k}^{1} \ln \left(s_{k}+r_{k}\right)\right)\right),
$$

where $s_{k}$ and $v_{k}$ are given by (8.4) and $r_{k}^{2}=s_{k}^{2}+4 v_{k} \bar{v}_{k}$.

In the situation of Theorem 7.1, restricting (8.5) to $U_{\Delta}$ and then to $X_{\Delta}$, that is, the subset of $U_{\Delta}$ where $v_{1}=\ldots=v_{d}=0$, gives the formula of Guillemin [Gu1] for the Kähler form of the toric variety $X_{\Delta}$. 


\section{The metric and generalized monopoles.}

Pedersen and Poon [PP] have given an explicit formula for the metric of a hyperkähler $4 n$-manifold $M$ with a free action of $T^{n}$ preserving the hyperkähler structure. Using the coordinate system $a_{i}, b_{i}$, they find that if $F$ is the function of Theorem 8.1 for $M$, then by putting

$$
\left(\Phi_{i j}, A_{j}\right)=\left(2 F_{a_{i} a_{j}}, \sum_{l} \sqrt{-1}\left(F_{a_{j} b_{l}} d b_{l}-F_{a_{j} \bar{b}_{l}} d \bar{b}_{l}\right)\right)
$$

we obtain a solution to the generalized Bogomolny equations with gauge group $T^{n}$. We call such a solution a monopole. More precisely, we can define a pair $(A, \Phi)$ by putting $A=\left(A_{1}, \ldots, A_{n}\right)$ and $\Phi=\left(\Phi_{1}, \ldots, \Phi_{n}\right)$ where $\Phi_{i}=\left(\Phi_{i 1}, \ldots, \Phi_{i n}\right)$. Then $A$ is a 1 -form on $\mathbb{R}^{3} \otimes \mathbb{R}^{n}$ with values in $\mathbb{R}^{n}$ and $\Phi_{i}$ are Higgs fields $\mathbb{R}^{3} \otimes \mathbb{R}^{n} \rightarrow \mathbb{R}^{n}$. If we put $w_{j}^{1}=a_{j}, w_{j}^{2}=\operatorname{Re} b_{j}, w_{j}^{3}=\operatorname{Im} b_{j}$, then $(A, \Phi)$ satisfy the linear system of PDEs

$$
\begin{aligned}
& R_{w_{i}^{\alpha} w_{j}^{\beta}}=\sum_{\gamma} \epsilon_{\alpha \beta \gamma} \nabla_{w_{i}^{\gamma}} \Phi_{j}, \\
& \nabla_{w_{i}^{\alpha}} \Phi_{j}=\nabla_{w_{j}^{\beta}} \Phi_{i}
\end{aligned}
$$

where $\epsilon$ is the alternating symbol, $\nabla=d+A$ is a connection on the trivial $\mathbb{R}^{n}$ bundle over $\mathbb{R}^{3} \otimes \mathbb{R}^{n}$ and $R$ is its curvature.

The hyperkähler metric $g$ on $M$ is given by

$$
g=-\sum_{i, j}\left[\Phi_{i j}\left(d a_{i} d a_{j}+d b_{i} d \bar{b}_{j}\right)+\Phi_{i j}^{-1}\left(d y_{i}+A_{i}\right)\left(d y_{j}+A_{j}\right)\right]
$$

where $d y_{i}=\sqrt{-1}\left(\bar{\partial}_{1} F_{a_{i}}-\partial_{1} F_{a_{i}}\right)$ are the fiber coordinates given by Killing vector fields corresponding to the action of $T^{n}$.

We shall now find the monopole corresponding to the metric on the toric hyperkähler manifold $M=M(\underline{u}, \underline{\lambda})$. We have to calculate partial derivatives $F_{a_{i} a_{j}}, F_{a_{i} b_{j}}, F_{a_{i} \bar{b}_{j}}$ where $F$ is given by (8.2) and (8.4). We have

$$
F_{a_{i}}=\sum_{k=1}^{d} \frac{\partial F}{\partial s_{k}} \frac{\partial s_{k}}{\partial a_{i}}=-\frac{1}{2} \sum_{k=1}^{d} \ln \left(s_{k}+r_{k}\right)\left(u_{k}\right)_{i}
$$

and then

$$
F_{a_{i} a_{j}}=-\sum_{k=1}^{d} \frac{\frac{\partial s_{k}}{\partial a_{j}}+\frac{\partial r_{k}}{\partial a_{j}}}{s_{k}+r_{k}}\left(u_{k}\right)_{i}=-\sum_{k=1}^{d} \frac{\left(u_{k}\right)_{j}\left(u_{k}\right)_{i}}{r_{k}}
$$


The geometry and topology of toric hyperkähler manifolds

$$
F_{a_{i} b_{j}}=-\frac{1}{2} \sum_{k=1}^{d} \frac{\frac{\partial r_{k}}{\partial b_{j}}}{s_{k}+r_{k}}\left(u_{k}\right)_{i}=-\sum_{k=1}^{d} \frac{\bar{v}_{k}\left(u_{k}\right)_{j}\left(u_{k}\right)_{i}}{\left(s_{k}+r_{k}\right) r_{k}}
$$

and

$$
F_{a_{i} \bar{b}_{j}}=-\sum_{k=1}^{d} \frac{v_{k}\left(u_{k}\right)_{j}\left(u_{k}\right)_{i}}{\left(s_{k}+r_{k}\right) r_{k}} .
$$

This gives us the monopole and therefore the following explicit formula for the metric on $M$ in terms of the moment map.

Theorem 9.1. On the open dense subset where the action of $T^{n}$ is free, the hyperkähler metric $g$ on $M$ is given by (9.3), where

$$
\left(\Phi_{i j}, A_{j}\right)=\left(-2 \sum_{k=1}^{d} \frac{\left(u_{k}\right)_{j}\left(u_{k}\right)_{i}}{r_{k}}, \sqrt{-1} \sum_{l=1}^{n} \sum_{k=1}^{d} \frac{\left(u_{k}\right)_{j}\left(u_{k}\right)_{l}}{\left(s_{k}+r_{k}\right) r_{k}}\left(v_{k} d \bar{b}_{l}-\bar{v}_{k} d b_{l}\right)\right),
$$

and $\frac{\partial}{\partial y_{j}}$ are infinitesimal isometries given by

$$
d y_{i}=\frac{\sqrt{-1}}{2}\left(\partial_{1}-\bar{\partial}_{1}\right) \sum_{k=1}^{d} \ln \left(s_{k}+r_{k}\right)\left(u_{k}\right)_{i}
$$

Once more, in the situation of Theorem 7.1 restricting to $v_{k}=0$ gives a formula for the Kähler metric on the toric variety $X_{\Delta}$.

Acknowledgements. We thank Krzysztof Galicki, Andy Nicas and Greg Sankaran for comments and conversations. We are particularly grateful to Jarosław Wiśniewski for explaining to us the rudiments of Mori's theory and for suggesting its use in the proof of Theorem 7.2. R.B. wishes to thank both McMaster University and the Max-Planck-Institut für Mathematik for hospitality and financial support. A.S.D. thanks NSERC for financial support.

\section{References.}

[BGM1] C.P. Boyer, K. Galicki and B.M. Mann, Quaternionic reduction and Einstein manifolds, Communications in Analysis and Geometry, 1 (1993), 229-279.

[BGM2] C.P. Boyer, K. Galicki and B.M. Mann, The geometry and topology of 3-Sasakian manifolds, J. Reine Angew. Math. 455 (1994), 183-220. 
[BGMR] C.P. Boyer, K. Galicki, B.M. Mann and E.G. Rees, Compact 3-Sasakian 7-manifolds with arbitrary second Betti number, Invent. Math 131 (1998), 321-344.

[Ca] E. Calabi, Métriques kählériennes et fibrés holomorphes, Ann. Éc. Norm. Sup. 12 (1979), 269-294.

[De] T. Delzant, Hamiltoniens périodiques et images convexe de l'application moment, Bull. Soc. Math. France 116 (1988), 315-339.

[Fu] W. Fulton, Introduction to toric varieties, Ann. Math. Studies, 131, Princeton University Press, Princeton (1993).

[GH] G.W. Gibbons and S.W. Hawking, Gravitational multi-instantons, Phys. Lett. 78B (1978), 430-432.

[GR] G.W. Gibbons and P. Rychenkova, HyperKähler quotient construction of BPS monopole moduli spaces, Cambridge DAMTP Commun. Math. Phys. 186 (1997), 581-599.

[Go] R. Goto, On toric hyper-Kähler manifolds given by the hyper-Kähler quotient method, in: Infinite Analysis (Kyoto 1991) part A, Adv. Ser. Math. Phys. 16, World Scientific, (1992).

[Gu1] V. Guillemin, Kähler structures on toric varieties, J. Diff. Geom. 40 (1994), 285-309.

[Gu2] V. Guillemin, Moment maps and combinatorial invariants of Hamiltonian $T^{n}$-spaces, Birkhäuser, Boston (1994).

[Hi] N.J. Hitchin, Polygons and gravitons, Math. Proc. Camb. Phil. Soc. 85 (1979), 465-476.

[HKLR] N.J. Hitchin, A. Karlhede, U. Lindström and M. Roček, Hyperkähler metrics and supersymmetry, Commun. Math. Phys. 108 (1987), 535589.

[Kr] P.B. Kronheimer, The construction of ALE spaces as hyper-Kähler quotients, J. Diff. Geom. 29 (1989), 665-683.

[Na] H. Nakajima, Instantons on ALE spaces, quiver varieties, and KacMoody algebras, Duke Math. J. 76 (1994), 365-416.

[Od] T. Oda, Convex bodies and algebraic geometry: an introduction to the theory of toric varieties, Springer, Heidelberg (1988).

[PP] H. Pedersen and Y.S. Poon, Hyper-Kähler metrics and a generalization of the Bogomolny equations, Comm. Math. Phys. 117 (1988), 569-580. 
[Re] M. Reid, Decomposition of toric morphisms, in Arithmetic and geometry. Papers dedicated to I.R. Shafarevich on the occasion of his sixtieth birthday, vol. II, Progress in Mathematics vol. 36, Birkhäuser, Boston (1983).

RECEIVED November 11, 1996.

Department of MATHEMATics, UNIVERSITY OF GLASGOW, GLASGOW, G12 8QW, UNITED KINGDOM

AND

Jesus College, OXFORD UNIVERSITY, OXFORD, OX1 3DW, UNITED KINGDOM

E-mail address: r.bielawski@maths.gla.ac.uk dancer@maths.ox.ac.uk 